DRAFt VERSION July 22, 2018

Preprint typeset using IAT $_{\mathrm{E}} \mathrm{X}$ style emulateapj v. 03/07/07

\title{
THE (BLACK HOLE MASS)-(HOST SPHEROID LUMINOSITY) RELATION AT HIGH AND LOW MASSES, THE QUADRATIC GROWTH OF BLACK HOLES, AND INTERMEDIATE-MASS BLACK HOLE CANDIDATES
}

\author{
Alister W. Graham ${ }^{1}$ and Nicholas Scott \\ Centre for Astrophysics and Supercomputing, Swinburne University of Technology, Hawthorn, Victoria 3122, Australia. \\ Draft version July 22, 2018
}

\section{ABSTRACT}

From a sample of 72 galaxies with reliable supermassive black hole masses $M_{\mathrm{bh}}$, we derive the $M_{\mathrm{bh}}-$ (host spheroid luminosity, $L$ ) relation for $i$ ) the subsample of 24 core-Sérsic galaxies with partially depleted cores, and ii) the remaining subsample of 48 Sérsic galaxies. Using $K_{s}$-band Two Micron All Sky Survey data, we find the near-linear relation $M_{\mathrm{bh}} \propto L_{\mathrm{K}_{\mathrm{s}}}^{1.10 .20}$ for the core-Sérsic spheroids thought to be built in additive dry merger events, while $M_{\mathrm{bh}} \propto L_{\mathrm{K}_{\mathrm{s}}}^{2.73 \pm 0.55}$ for the Sérsic spheroids built from gas-rich processes. After converting literature $B$-band disk galaxy magnitudes into inclinationand dust-corrected bulge magnitudes, via a useful new equation presented herein, we obtain a similar result. Unlike with the $M_{\mathrm{bh}}$-(velocity dispersion) diagram, which is also updated here using the same galaxy sample, it remains unknown whether barred and non-barred Sérsic galaxies are offset from each other in the $M_{\mathrm{bh}}-L$ diagram.

While black hole feedback has typically been invoked to explain what was previously thought to be a nearly constant $M_{\mathrm{bh}} / M_{\text {Spheroid }}$ mass ratio of $\sim 0.2 \%$, we advocate that the near-linear $M_{\mathrm{bh}}{ }^{-}$ $L$ and $M_{\mathrm{bh}}-M_{\text {Spheroid }}$ relations observed at high masses may have instead largely arisen from the additive dry merging of galaxies. We argue that feedback results in a dramatically different scaling relation, such that black hole mass scales roughly quadratically with the spheroid mass in Sérsic galaxies. We therefore introduce a revised cold-gas 'quasar' mode feeding equation for semi-analytical models to reflect what we dub the quadratic growth of black holes in Sérsic galaxies built amidst gas-rich processes. Finally, we use our new Sérsic $M_{\mathrm{bh}}-L$ equations to predict the masses of candidate intermediate mass black holes in almost 50 low luminosity spheroids containing active galactic nuclei, finding many masses between that of stellar mass black holes and supermassive black holes.

Subject headings: black hole physics — galaxies: evolution — galaxies: nuclei

\section{INTRODUCTION}

The (black hole mass)-(host spheroid dynamical mass) $M_{\mathrm{bh}}-M_{\mathrm{Sph}, \mathrm{dyn}}$ relation (e.g. Marconi \& Hunt 2003; Häring \& Rix 2004) has recently been revised such that core-Sérsic galaxies (whose spheroidal components are thought to be built in simple additive dry merger events) define a near-linear relation while Sérsic galaxies (whose spheroidal components are likely built amidst gas-rich processes) define a near-quadratic relation (Graham 2012a). Graham (2012a,b) additionally predicted that the previously established log-linear, i.e. single power-law, relation between black hole mass and host spheroid luminosity, $L$, must also require a significant modification such that $M_{\mathrm{bh}} \propto L^{1.0}$ for the spheroidal component of luminous $\left(M_{B} \lesssim-20.5 \mathrm{mag}\right)$ core-Sérsic galaxies while $M_{\mathrm{bh}} \propto L^{2.5}$ for the fainter Sérsic spheroids - if their dynamical mass-to-light ratio scales with $L^{1 / 4}$. If $(M / L)_{\text {dynamical }} \propto L^{1 / 3}$ then one expects $M_{\mathrm{bh}} \propto L^{8 / 3}$. Here, for the first time, we show that the $M_{\mathrm{bh}}-L$ relation is indeed bent, and we provide new expressions to predict black hole masses in core-Sérsic galaxies and Sérsic galaxies using the luminosity of their spheroidal component. $^{2}$

From a literature sample of eight galaxies, Kormendy

\footnotetext{
${ }^{1}$ Corresponding Author: AGraham@swin.edu.au

${ }^{2}$ Readers unfamiliar with the core-Sérsic model (Graham et al. 2003; Trujillo et al. 2004) or the Sérsic (1963) model, for the classification of galaxies, may like to refer to the review article by Graham \& Driver (2005).
}

\& Richstone (1995, their figure 14) revealed an apparently linear correlation between black hole mass and the brightness / mass of the host spheroid. As the sample size grew, researchers began to apply a single log-linear, i.e. power-law, relation to the data (e.g. Richstone et al. 1998; Kormendy \& Gebhardt 2001; McClure \& Dunlop 2002). However the samples remained dominated by fairly luminous spheroids and the applicability of the new $M_{\mathrm{bh}}-L$ relations (e.g. McClure \& Dunlop 2004; Marconi \& Hunt 2003; Graham 2007a) to low-luminosity systems was not securely established. Indeed, Graham (2007a) noted that the $M_{\mathrm{bh}}-L$ and $M_{\mathrm{bh}}-\sigma$ relations (Ferrarese \& Merritt 2000; Gebhardt et al. 2000) cannot both be described by a power-law because the relationship between luminosity and velocity dispersion, $\sigma$, for elliptical galaxies is not described by a single power-law.

As reviewed in Graham (2012c), the elliptical galaxy $L-\sigma$ relation has long been known to be 'broken', such that $L \propto \sigma^{5}$ at the luminous-end $\left(M_{B} \lesssim-20.5\right.$ mag: Schechter 1980; Malumuth \& Kirshner 1981; Von Der Linden et al. 2007; Liu et al. 2008) and possibly even as steep as $L \propto \sigma^{6}$ (Lauer et al. 2007), while $L \propto \sigma^{2}$ at intermediate and faint luminosities (e.g. Davies et al. 1983; Held et al. 1992; de Rijcke et al. 2005; Matković \& Guzmán 2005; Kourkchi et al. 2012). Galaxy samples containing both bright and intermediate luminosity elliptical galaxies will therefore naturally yield $L-\sigma$ relationships with exponents around 3 to 4 (e.g., Faber \& Jackson 1976; Tonry 1981; de Vaucouleurs \& Olson 
1982; Desroches et al. 2007). It should be noted that this change in slope of the $L-\sigma$ relation at $M_{B} \approx-20.5 \mathrm{mag}$ (Sérsic $n \approx 3-4$ ) is not related to (i) the onset of the coexistence of classical bulges and pseudobulges at $n_{\text {bulge }} \lesssim 2$, nor (ii) the alleged divide at $M_{B}=-18 \mathrm{mag}\left(n_{\text {galaxy }} \lesssim\right.$ 1-2) between dwarf and ordinary elliptical galaxies (Kormendy 1985; Kormendy et al. 2009). Instead, the change in slope at $M_{B} \approx-20.5 \mathrm{mag}$ coincides with the division between core-Sérsic galaxies (which have a central deficit of light relative to the inward extrapolation of their outer Sérsic light profile) and Sérsic galaxies ${ }^{3}$ which do not (Graham \& Guzmán 2003; Graham et al. 2003; Trujillo et al. 2004; Matković \& Guzmán 2005). If (non-barred) Sérsic and core-Sérsic galaxies follow the same $M_{\mathrm{bh}} \propto \sigma^{5}$ relation (see section 3.1), they therefore obviously cannot follow the same $M_{\mathrm{bh}}-L$ relation because they do not follow the same $L-\sigma$ relation. Simple consistency arguments dictate that the core-Sérsic galaxies should follow the relation $M_{\mathrm{bh}} \propto L^{1.0}$ while the (non-barred) Sérsic galaxies should follow the relation $M_{\mathrm{bh}} \propto L^{2.5}$ (or $M_{\mathrm{bh}} \propto L^{3}$ if $\left.M_{\mathrm{bh}} \propto \sigma^{6}\right)$.

While the core-Sérsic model has provided a new means to identify and quantify the stellar deficits, i.e. the flattened cores, which have long been observed in giant galaxies (e.g. King 1978, and references therein), the rationale for a physical divide between core-Sérsic galaxies and Sérsic galaxies also existed long ago. Early-type galaxies brighter than $M_{B}=-20.5 \mathrm{mag}$ tend to be anisotropic, pressure supported elliptical galaxies with boxy isophotes, while the less luminous early-type galaxies tend to have disky isophotes and often contain a rotating disk of stars (e.g. Carter 1978, 1987; Davies et al. 1983; Bender et al. 1988; Peletier et al. 1990; Jaffe et al. 1994; Faber et al. 1997; Emsellem et al. 2011). In addition, and as noted already, the $L-\sigma$ relation changes slope at $M_{B} \approx-20.5 \mathrm{mag}$ (Davies et al. 1983; Matković \& Guzmán 2005): the core-Sérsic galaxies $\left(M_{B} \lesssim-20.5\right.$ mag) define the relation $L \propto \sigma^{5}$ while the Sérsic galaxies $\left(M_{B} \gtrsim-20.5 \mathrm{mag}\right)$ follow the relation $L \propto \sigma^{2}$, with no discontinuity at $M_{B}=-18$ mag. Similarly, the elliptical galaxy $L-\mu_{0}$ relation, where $\mu_{0}$ is the central surface brightness, also breaks at $M_{B} \approx-20.5 \mathrm{mag}$ and remains linear at $M_{B}=-18 \mathrm{mag}$ (e.g. Graham \& Guzmán 2003 $)^{4}$. While gas-rich processes and mergers are thought to build the Sérsic galaxies, relatively gasfree galaxy mergers are thought to build the core-Sérsic galaxies. The coalescence of massive black holes - from the pre-merged, gas-poor galaxies - scour out the core of the newly merged 'core-Sérsic' galaxy (Begelman, Blandford, \& Rees 1980; Ebisuzaki, Makino, \& Okumura 1991; Merritt, Mikkola, \& Szell 2007).

The above expectation for the 'bent' $M_{\mathrm{bh}}-L$ relation impacts dramatically upon our predictions for black hole masses at the centers of other galaxies, has implications for the competing formation scenarios proposed to explain the coevolution of galactic spheroids and their su-

\footnotetext{
${ }^{3}$ Sérsic galaxies were previously referred to as 'power-law' galaxies before it was realized that their spheroidal component's inner light profiles are better described by the curved Sérsic model than by a power-law (e.g. Trujillo et al. 2004).

4 As shown, and explained, in Graham 2012c, it is only from the use of 'effective' radii and 'effective' surface brightnesses that apparent breaks are seen at $M_{B}=-18 \mathrm{mag}$ in what are actually continuous, curved relations.
}

permassive black holes, and has ramifications for semianalytic computer codes which try to mimic this coevolution. Moreover, as noted in Graham (2012a), the realization of a broken $M_{\mathrm{bh}}-L$ relation will influence, among other things: evolutionary studies of the (black hole)(host spheroid) connection over different cosmic epochs (e.g. Cisternas et al. 2011; Li, Ho \& Wang 2011; Hiner et al. 2012; Zhang, Lu \& Yu 2012); predictions for spacebased gravitational wave detections which use the $M_{\mathrm{bh}}-L$ relation to estimate black hole masses (e.g. Mapelli et al. 2012, and references therein); and estimates of the black hole mass function and mass density based on spheroid luminosity functions (e.g. Vika et al. 2009; Li, Wang, \& Ho 2012).

Recent studies of the $M_{\mathrm{bh}}-L$ relation have continued to be largely dominated by luminous spheroids with $M_{\mathrm{bh}} \gtrsim 10^{7}-10^{8} M_{\odot}$, and have continued to fit a straight line to the data (e.g. Sani et al. 2011; Vika et al. 2011; McConnell et al. 2011a; Beifiori et al. 2012). However, an inspection of their $M_{\mathrm{bh}}-L$ diagrams reveals the onset of a steepening relation in their lower luminosity spheroids which have $M_{\mathrm{bh}} \lesssim 10^{7}-10^{8} M_{\odot}$, as also seen in the diagrams from Graham (2007a) and Gültekin et al. (2009b). In addition, a number of models are actually generating 'bent' $M_{\mathrm{bh}}-M_{\text {Spheroid }}$ relations which show a steepening at low masses (e.g. Cirasuolo et al. 2005, their figure 5; Khandai et al. 2012, their figure 7; Bonoli, Mayer \& Callegari 2012, their figure 7). Here we build on this (tentative observational) evidence of a steepening relation at low masses by using a sample of 72 galaxies with directly measured black hole masses that are tabulated in Section 2 and $\operatorname{span} 10^{6}-10^{10} M_{\odot}$. For the first time we both identify and quantify the bend in the $M_{\mathrm{bh}}-L$ relation (Section 3).

Rather than fitting a single quadratic relation to the $\left(M_{\mathrm{bh}}, L\right)$ distribution of points - which would be in line with the use of a log-quadratic relation in the $M_{\mathrm{bh}}-n$ diagram by Graham \& Driver (2007a) — we embrace the large body of data which shows that Sérsic and coreSérsic galaxies have distinguishing characteristics suggestive of a different formation history (e.g. Davies et al. 1983; Faber et al. 1997; Graham \& Guzmán 2003; Gavazzi et al. 2005; Ferrarese et al. 2006) and therefore fit two $M_{\mathrm{bh}}-L$ relations, one for each type of galaxy.

In Section 4 we provide a discussion of some of the more salient points arising from the new $M_{\mathrm{bh}}-L$ relation for Sérsic and core-Sérsic galaxies. In particular, Section 4.1 provides updated black hole mass estimates for nearly 50 low-luminosity spheroids, and reveals that they occupy the holy grail mass range for intermediate mass black holes, ranging from the stellar mass black hole upper limit of $\sim 10^{2} M_{\odot}$ to the lower-bound of $10^{5}-10^{6} M_{\odot}$ for supermassive black holes. In Section 4.2 we introduce a significantly modified expression for use in semianalytical models which try to mimic the coevolution of supermassive black holes and galaxies. We provide a new quadratic cold-gas 'quasar' mode feeding equation to match the quadratic (black hole)-to-(host spheroid) growth observed in Sérsic galaxies built from gas-rich processes. Finally, Section 4.3 discusses the observation that barred and non-barred disk galaxies currently appear to occupy the same region of the $M_{\mathrm{bh}}-L$ diagram. 
We have continued to build on past catalogs of reliable supermassive black hole masses obtained from direct maser, stellar or gas kinematic measurements (e.g. Ferrarese \& Ford (2005); Hu (2008); Graham (2008b); Graham et al. 2011) by adding 16 galaxies from the literature. This gives us an initial sample of 80 galaxies with directly measured black hole masses. Since the catalog in Graham et al. (2011) was prepared, we note that several updates over the last two years have resulted in the doubling of black hole masses at the high-mass end of the $M_{\mathrm{bh}}-\sigma$ diagram (e.g. van den Bosch \& de Zeeuw 2010; Walsh et al. 2010, 2012) and two ten-billion solar mass black holes have been reported (e.g. McConnell et al. 2012).

\subsection{Galaxy exclusions}

Separate from the above sample of 80 galaxies, we have maintained our exclusion of NGC 7457, because its black hole mass was derived assuming that this galaxy's excess nuclear light was due to an AGN rather than a massive nuclear disk and dense star cluster (Balcells et al. 2007; Graham \& Spitler 2009), and the exclusion of the Sc galaxy NGC 2748 due to potential dust complications with its estimated black hole mass (Atkinson et al. 2005; $\mathrm{Hu} 2008$ ).

For the following reasons, our sample of 80 galaxies is reduced here by five. The best-fitting parameter set in the mass modeling of NGC 5252 by Capetti et al. (2005) had resulted in a reduced $\chi^{2}$ value of 16.5 , indicative of a poor fit to the data and resulting in its exclusion by Gültekin et al. (2009b). We too now exclude this galaxy, which would otherwise appear to have a black hole mass that is an order of magnitude too high in the $M_{\mathrm{bh}}-\sigma$ diagram. The inclined, starburst spiral galaxy Circinus is also excluded due to its complex kinematics (e.g. For, Koribalski \& Jarrett 2012, and references therein) and location on the other side of the Galactic plane. With a Galactic latitude of less than 5 degrees, the suggested $B$ band extinction correction for Circinus is 5 mag or more (Schlafly \& Finkbeiner 2011) and is considered to be unreliable. The above two galaxies can not be used reliably in either the $M_{\mathrm{bh}}-L$ diagram or the $M_{\mathrm{bh}}-\sigma$ diagram, and they are therefore not listed in our Table 1.

Following Gültekin et al. (2009b), we exclude the barred galaxy NGC 3079. Although NGC 3079 has a well determined black hole mass (Kondratko et al. 2005), the observed stellar velocity dispersion drops rapidly from a central value of $\sim 150 \mathrm{~km} \mathrm{~s}^{-1}$ to $60 \mathrm{~km} \mathrm{~s}^{-1}$ or less within just $1 \mathrm{kpc}$ (Shaw, Wilkinson, \& Carter 1993). The dynamics in this Sc galaxy's boxy peanut-shaped bulge are known to be affected by bar streaming motions (Merrifield \& Kuijken (1999; Veilleux, Bland-Hawthorn, \& Cecil 1999) which were not modeled by Shaw et al. (1993). Such additional motions are a general concern in barred galaxies, as detailed in Graham et al. (2011), and it is not clear what velocity dispersion should be used for the bulge component of this galaxy ${ }^{5}$. The bulge luminosity of the Milky Way is uncertain due to dust in our Galaxy's disc, and is therefore not included in our $M_{\mathrm{bh}}-L_{\text {spheroid }}$ diagram. Finally, we also exclude M32 which belongs to

\footnotetext{
${ }^{5}$ While this galaxy could be used in the $M_{\mathrm{bh}}-L_{\text {spheroid }}$ diagram, we do not so as to be consistent with our galaxy sample in the final $M_{\mathrm{bh}}-\sigma$ diagram.
}

the rare 'compact elliptical' galaxy class and is thus not representative of the majority of galaxies. Such galaxies are thought to be heavily stripped of an unknown fraction of their stars (e.g. Bekki et al. 2001), and as such this galaxy may bias our analysis. We do however note that M32, and the Milky Way, have been included in a preliminary $M_{\mathrm{bh}}-\sigma$ diagram (Figure 1). We therefore mention here that we have used a host bulge velocity dispersion of $55 \mathrm{~km} \mathrm{~s}^{-1}$ from M32's bulge stars outside of M32's core region; this value is lower than the commonly used central aperture values of $72-75 \mathrm{~km} \mathrm{~s}^{-1}$ which are biased high by the dynamics around the central black hole (Igor Chilingarian, priv. comm.). This leaves us, thus far, with a sample of $(80-5=) 75$ galaxies. For reasons discussed at the start of Section 3 , an additional three galaxies (NGC 1316, NGC 3842 and NGC 4889) are excluded from some of the analyses.

\subsection{Galaxy distances and magnitudes}

For the bulk of the sample, we have used the distance moduli from Tonry et al. (2001) after first decreasing these values by $0.06 \mathrm{mag}$ and thereby reducing both the galaxy distances and the black hole masses by $\sim 3 \%$. This small adjustment arises from Blakeslee et al.'s (2002, their Section 4.6) recalibration of the surface brightness fluctuation method based on the final Cepheid distances given by Freedman et al. (2001, with the metallicity correction). In addition to listing each galaxy's distance and velocity dispersion, primarily taken from HyperLeda ${ }^{6}$ (Paturel et al. 2003), Table 1 presents their observed (i.e. uncorrected) $B$-band magnitude, $B_{T}$, from the Third Reference Catalogue of Bright Galaxies (de Vaucouleurs et al. 1991, hereafter RC3) and their total $K_{s}$-band magnitude as provided by the Two Micron All Sky Survey (2MASS) ${ }^{7}$ (Jarrett et al. 2000). Due to the bright star near the center of NGC 2974, we used the $K$-band magnitude from Cappellari et al. (2006) rather than the 2MASS value for this galaxy.

Both the observed $B$ - and $K_{s}$-band magnitudes need to be corrected for Galactic extinction, which we have taken from Schlegel et al. (1998), as provided by the NASA/IPAC Extragalactic Database (NED) ${ }^{8}$ and included in Table 1 as $A_{B}$ and $A_{K}$ for ease of reference. We additionally provide K-corrections for the 2MASS $K_{s}$-band magnitudes, derived from each galaxy's heliocentric redshift $z$ (taken from NED) and total $J-K$ color (taken from 2MASS) coupled with the prescription from Chilingarian, Melchior \& Zolotukhin (2010) which is available online ${ }^{9}$. In passing, we note that for the low redshifts associated with our galaxy sample, this $\mathrm{K}_{K_{s}}$ correction is approximately $-2.1 z,-2.2 z$, and $-2.5 z$ for the elliptical, S0 and Sa, and later spiral galaxy types, respectively. Without reliable $B-R$ or $B-I$ colors, the $B$-band K-corrections tabulated in Table 1 are such that $\mathrm{K}_{B}$ equals $4 z, 3.5 z, 3 z$, and $2.5 z$ for the elliptical, $\mathrm{S} 0$, $\mathrm{Sa}$, and later spiral galaxy types, respectively. As can be seen in Table 1, these corrections are minor and they have had no significant impact on the final results. Finally, in our analysis we corrected all galaxy magnitudes

\footnotetext{
6 http://leda.univ-lyon1.fr

7 www.ipac.caltech.edu/2mass

8 http://nedwww.ipac.caltech.edu

9 http://kcor.sai.msu.ru
} 
for cosmological redshift dimming.

\subsection{Bulge magnitudes}

In addition to the above three corrections that were applied in the analysis rather than in Table 1, the tabulated disk galaxy magnitudes were adjusted for two other factors: their observed, total magnitudes were converted into dust-corrected, bulge magnitudes. Rather than do this galaxy by galaxy (e.g. Grootes et al. 2012), which would require careful bulge/disk decompositions, we can take advantage of our large sample size and employ a mean statistical correction. While this will result in individual bulge magnitudes not being exactly correct, the ensemble average correction will be correct. Given that dust has an order of magnitude less impact in the $K_{s^{-}}$ band than in the $B$-band, we can expect there to be less scatter in our $K_{s}$-band $M_{\mathrm{bh}}-L$ relation, at least for the Sérsic sample which contains the bulk of the bulges.

Using the relation $M=-2.5 \log (L)$, the observed and the intrinsic (i.e. dust-corrected) bulge and disk luminosities are related as follows.

$$
\begin{gathered}
L_{\text {bulge }, \text { obs }} / L_{\text {bulge }, \text { int }}=10^{-\Delta M_{\text {bulge }} / 2.5}, \\
L_{\text {disk,obs }} / L_{\text {disk,int }}=10^{-\Delta M_{\text {disk }} / 2.5}, \\
L_{\text {bulge,obs }}+L_{\text {disk,obs }}=10^{-M_{\text {galaxy }, \text { obs }} / 2.5}, \\
L_{\text {bulge,int }} / L_{\text {disk,int }}=B / D,
\end{gathered}
$$

where $\Delta M_{\text {bulge }}$ and $\Delta M_{\text {disk }}$ are the differences between the observed and intrinsic magnitudes (given below). The above equations can be combined to give the useful expression

$$
\begin{aligned}
M_{\text {bulge }, \text { int }}= & M_{\text {galaxy }, \text { obs }} \\
& +2.5 \log \left[10^{-\Delta M_{\text {bulge }} / 2.5}+\frac{10^{-\Delta M_{\text {disk }} / 2.5}}{(B / D)}\right](5)
\end{aligned}
$$

The average dust-corrected bulge-to-disk $(B / D)$ flux ratio is well known to be a function of both galaxy morphological type and passband, varying mainly due to the bulge luminosity (e.g. Yoshizawa \& Wakamatsu 1975; Trujillo et al. 2001). Graham \& Worley (2008) presented over $400 K$-band, $B / D$ flux ratios as a function of disk galaxy morphological type. Given that extinction due to dust is still an issue at near-infrared wavelengths, albeit notably less in the $K$-band than the $H$-band, these flux ratios were corrected by Graham \& Worley (2008) for dust following the prescription given by Driver et al. (2008). Most studies have not made this important correction which effectively increases the observed $B / D$ flux ratio after accounting for the light from the far-side of the bulge which is obscured by the centrally-concentrated dust in the disk. Here we have slightly adjusted Graham \& Worley's (2008) $B / D$ values by combining the S0 and S0/a classes as one in our Table 2 and ensuring that they do not have a smaller $B / D$ ratio than the Sa galaxies. By combining these ratios with the average, inclinationdependent $\Delta M_{\text {bulge }}$ and $\Delta M_{\text {disk }}$ values below, one can derive the expected 'intrinsic' bulge magnitude from the observed (dust-dimmed) galaxy magnitude and the inclination of the disk. Specifically, for $\Delta M_{\text {bulge }}$ and $\Delta M_{\text {disk }}$ we have used the expressions from Driver et al. (2008) such that

$\Delta M_{\mathrm{bulge}}=M_{\mathrm{bulge}, \mathrm{obs}}-M_{\mathrm{bulge}, \text { intrin }}=b_{1}+b_{2}[1-\cos (i)]^{b_{3}}$, and

$\Delta M_{\mathrm{disk}}=M_{\mathrm{disc}, \mathrm{obs}}-M_{\mathrm{disc}, \text { intrin }}=d_{1}+d_{2}[1-\cos (i)]^{d_{3}}$,

where the coefficients in the above equations depend on the passband used and are provided in Driver et al. (2008). The cosine of the disk inclination angle $i$, such that $i=0$ degrees for a face-on disk, is roughly equal to the minor-to-major axis ratio, $b / a$, at large radii. For our galaxy sample, these values have been provided in the final column of Table 1 .

We have used a conservative (small) uncertainty of $5 \%$ for the velocity dispersions, and assigned a typical uncertainty of $0.25 \mathrm{mag}$ to the elliptical galaxy magnitudes. Regarding the disk galaxies, the observed range of bulgeto-disk flux ratios (Graham \& Worley 2008) has a $1 \sigma$ scatter equal to a factor of $\sim 2$ for any given disk galaxy morphological type. We therefore assign a notably larger uncertainty of $0.75 \mathrm{mag}$ to our bulge magnitudes. While this may at first sound worryingly high, we again note that the sample average shift from a disk galaxy magnitude to a bulge magnitude will be more accurate, thereby much less affecting the recovery of the $M_{\mathrm{bh}}-L$ relation. It is only now that the disk galaxy sample size $N=44$ is sufficiently large (coupled with 9 elliptical galaxies in the Sérsic sample) that we can use this methodology because the uncertainty in the disk-galaxy sample average correction scales with $1 / \sqrt{N}$.

\subsection{Galaxy core type}

As described in the Introduction, several global properties and scaling relations of galaxies depend on their core type. We have therefore identified galaxies with or without a partially depleted stellar core. Such cores, with shallow light profiles, are thought to have formed during 'dry' galaxy merger events in which a binary supermassive black hole's orbit decays by gravitationally ejecting stars from the center of the newly merged galaxy (e.g. Begelman et al. 1980; Ebisuzaki et al. 1991; Graham 2004; Merritt et al. 2007). However Sérsic galaxies that have $n \lesssim 2-3$ may also have a resolved inner, negative logarithmic light-profile slope (for the spheroid) which is less steep than 0.3, although they have no depleted core relative to their outer profile. Graham et al. (2003) warned that some of these galaxies may be misidentified as 'core' galaxies and Dullo \& Graham (2012) have shown that this has occurred $\sim 20 \%$ of the time in the past. We therefore have a preference for cores identified as a deficit relative to the inward extrapolation of the spheroid's outer Sérsic profile (e.g. Trujillo et al. 2004; Ferrarese et al. 2006; Dullo \& Graham 2012). Such galaxies are referred to as 'core-Sérsic' galaxies, while those with no deficit or instead additional nuclear components are referred to as 'Sérsic' galaxies (e.g. Graham et al. 2003; Graham \& Guzmán 2003; Balcells et al. 2003; Trujillo et al. 2004).

For most of the luminous galaxies we have been able to identify from suitably high-resolution images whether or not they possess a partially-depleted core, although some are at too great a distance to know. At the lowluminosity end of the relation, dusty nuclei can make this task more challenging. When no core designation was available or possible from the literature (see column 4 of Table 10 we used the velocity dispersion to help us 
The $M_{\mathrm{bh}}-L_{\mathrm{spheroid}}$ relation

TABLE 1

Black hole / Galaxy data.

\begin{tabular}{|c|c|c|c|c|c|c|c|c|c|c|c|c|c|c|}
\hline $\begin{array}{c}\text { Galaxy } \\
\text { (1) }\end{array}$ & $\begin{array}{c}\text { Type } \\
(2)\end{array}$ & $\begin{array}{c}\text { Dist } \\
{[\mathrm{Mpc}]} \\
(3)\end{array}$ & $\begin{array}{l}\text { core } \\
(4)\end{array}$ & $\begin{array}{c}\sigma \\
\mathrm{km} \mathrm{s}_{(5)}^{-1}\end{array}$ & $\begin{array}{c}M_{\mathrm{bh}} \\
{\left[10^{8} M_{\odot}\right]} \\
(6)\end{array}$ & $\begin{array}{c}B_{T} \\
{[\mathrm{mag}]} \\
(7)\end{array}$ & $\begin{array}{c}K_{s} \\
\underset{(8)}{[\mathrm{mag}]}\end{array}$ & $\begin{array}{c}\mathrm{A}_{B} \\
{[\mathrm{mag}]} \\
(9)\end{array}$ & $\begin{array}{c}\mathrm{A}_{K} \\
{[\mathrm{mag}]} \\
(10)\end{array}$ & $\begin{array}{c}\mathrm{K}_{B} \\
{[\mathrm{mag}]} \\
(11)\end{array}$ & $\begin{array}{c}\mathrm{K}_{K_{s}} \\
{[\mathrm{mag}]} \\
(12)\end{array}$ & $\begin{array}{l}\mathrm{R}_{25} \\
\text { (13) }\end{array}$ & $\begin{array}{c}\mathrm{M}_{B} \\
{[\mathrm{mag}]} \\
(14)\end{array}$ & $\begin{array}{c}\mathrm{M}_{K_{s}} \\
{[\mathrm{mag}]} \\
(15)\end{array}$ \\
\hline \multicolumn{15}{|c|}{30 Ellipticals } \\
\hline A1836, BCG & E1 & $158.0[3 \mathrm{a}]$ & $\mathrm{y} ?$ & $309[5 \mathrm{a}]$ & $39_{-5}^{+4}[6 \mathrm{a}]$ & 14.56 & 9.993 & 0.277 & 0.024 & 0.15 & -0.07 & $\ldots$ & -21.72 & -26.25 \\
\hline A3565, BCG & E1 & $40.7[3 a]$ & $\mathrm{y} ?$ & $335[5 \mathrm{~b}]$ & $11_{-2}^{+2}[6 \mathrm{a}]$ & 11.61 & 7.502 & 0.265 & 0.023 & 0.05 & -0.03 & $\ldots$ & -21.71 & -25.65 \\
\hline IC 1459 & E3 & 28.4 & $\mathrm{y} ?$ & 306 & $24_{-10}^{+10}[6 \mathrm{~b}]$ & 11.61 & 7.502 & 0.265 & 0.023 & 0.02 & -0.01 & $\ldots$ & -21.37 & -25.50 \\
\hline NGC 821 & E6 & 23.4 & $\mathrm{n}[4 \mathrm{a}]$ & 200 & $0.39_{-0.09}^{+0.26}[6 \mathrm{c}]$ & 11.67 & 7.900 & 0.474 & 0.040 & 0.02 & -0.01 & ... & -20.65 & -24.02 \\
\hline NGC 1399 & E1 & 19.4 & $\mathrm{y}[4 \mathrm{~b}]$ & 329 & $4.7_{-0.6}^{+0.6}[6 \mathrm{~d}]$ & 10.55 & 6.306 & 0.056 & 0.005 & 0.02 & -0.01 & $\ldots$ & -20.95 & -25.17 \\
\hline NGC 2974 & $\mathrm{E} 4$ & 20.9 & $\mathrm{n}[4 \mathrm{c}]$ & 227 & $1.7_{-0.2}^{+0.2}[6 \mathrm{e}]$ & 11.87 & $8.00[8 \mathrm{a}]$ & 0.235 & 0.020 & 0.03 & -0.01 & $\ldots$ & -19.97 & -23.66 \\
\hline NGC 3377 & E5 & 10.9 & $\mathrm{n}[4 \mathrm{~d}]$ & 139 & $0.77_{-0.06}^{+0.04}[6 \mathrm{f}]$ & 11.24 & 7.441 & 0.147 & 0.013 & 0.01 & -0.01 & $\ldots$ & -19.09 & -22.78 \\
\hline NGC 3379 & E1 & 10.3 & $\mathrm{y}[4 \mathrm{~b}]$ & 209 & $4.0_{-1.0}^{+1.0}[6 \mathrm{~g}]$ & 10.24 & 6.270 & 0.105 & 0.009 & 0.01 & -0.01 & $\ldots$ & -19.93 & -23.83 \\
\hline NGC 3608 & $\mathrm{E} 2$ & 22.3 & $\mathrm{y}[4 \mathrm{~b}]$ & 192 & $2.0_{-0.6}^{+1.1}[6 \mathrm{c}]$ & 11.70 & 8.096 & 0.090 & 0.008 & 0.02 & -0.01 & $\ldots$ & -20.13 & -23.68 \\
\hline NGC 3842 & E3 & $98.4[6 \mathrm{~h}]$ & $\mathrm{y}[4 \mathrm{~b}]$ & $270[6 \mathrm{~h}]$ & $97_{-26}^{+30}[6 \mathrm{~h}]$ & 12.78 & 9.082 & 0.093 & 0.008 & 0.08 & -0.04 & $\ldots$ & -22.28 & -26.02 \\
\hline NGC 4261 & $\mathrm{E} 2$ & 30.8 & $\mathrm{y}[4 \mathrm{e}]$ & 309 & $5.0_{-1.0}^{+1.0}[6 \mathrm{i}]$ & 11.41 & 7.263 & 0.078 & 0.007 & 0.03 & -0.02 & ... & -21.11 & -25.24 \\
\hline NGC 4291 & E3 & 25.5 & $\mathrm{y}[4 \mathrm{~b}]$ & 285 & $3.3_{-2.5}^{+0.9}[6 \mathrm{c}]$ & 12.43 & 8.417 & 0.158 & 0.013 & 0.02 & -0.01 & $\ldots$ & -19.76 & -23.66 \\
\hline NGC 4374 & E1 & 17.9 & $\mathrm{y}[4 \mathrm{f}]$ & $296[6 \mathrm{j}]$ & $9.0_{-0.8}^{+0.9}[6 \mathrm{j}]$ & 10.09 & 6.222 & 0.174 & 0.015 & 0.01 & -0.01 & ... & -21.35 & -25.08 \\
\hline NGC 4473 & E5 & 15.3 & $\mathrm{n}[4 \mathrm{~b}]$ & 179 & $1.2_{-0.9}^{+0.4}[6 \mathrm{c}]$ & 11.16 & 7.157 & 0.123 & 0.010 & 0.03 & -0.02 & ... & -19.89 & -23.83 \\
\hline NGC 4486 & E1 & 15.6 & $\mathrm{y}[4 \mathrm{f}]$ & 334 & $58_{-3.5}^{+3.5}[6 \mathrm{k}]$ & 09.59 & 5.812 & 0.096 & 0.008 & 0.02 & -0.01 & $\ldots$ & -21.47 & -25.19 \\
\hline NGC $4486 a$ & $\mathrm{E} 2$ & $17.0[3 \mathrm{~b}]$ & $\mathrm{n}[4 \mathrm{f}]$ & $110[5 \mathrm{c}]$ & $0.13_{-0.08}^{+0.08}[61]$ & $13.20[7 \mathrm{a}]$ & 9.012 & 0.102 & 0.009 & 0.00 & -0.00 & $\ldots$ & -18.05 & -22.15 \\
\hline NGC 4552 & E0 & 14.9 & $\mathrm{y}[4 \mathrm{~b}]$ & 252 & $4.7_{-0.5}^{+0.5}[6 \mathrm{e}]$ & 10.73 & 6.728 & 0.177 & 0.015 & 0.00 & -0.00 & $\ldots$ & -20.31 & -24.16 \\
\hline NGC 4621 & E5 & 17.8 & $\mathrm{n}[4 \mathrm{f}]$ & 225 & $3.9_{-0.4}^{+0.4}[6 \mathrm{e}]$ & .57 & 746 & 0.143 & 0.012 & 0.01 & -0.00 & $\ldots$ & -20.83 & -24.52 \\
\hline NGC 4649 & E2 & 16.4 & $\mathrm{y}[4 \mathrm{~b}]$ & 335 & $47_{-10}^{+10}[6 \mathrm{~m}]$ & 9.81 & 5.739 & 0.114 & 0.010 & 0.01 & -0.01 & $\ldots$ & -21.38 & -25.37 \\
\hline NGC 4697 & E6 & 11.4 & $\mathrm{n}[4 \mathrm{~g}]$ & 171 & $1.8_{-0.1}^{+0.2}[6 \mathrm{c}]$ & 10.14 & 6.367 & 0.131 & 0.011 & 0.02 & -0.01 & . & -20.28 & -23.96 \\
\hline NGC 4889 & $\mathrm{E} 4$ & $103.2[6 \mathrm{~h}]$ & $\mathrm{y}[4 \mathrm{~g}]$ & $347[6 \mathrm{~h}]$ & $210_{-160}^{+160}[6 \mathrm{~h}]$ & 12.53 & 407 & 0.041 & 0.004 & 0.09 & -0.04 & ... & -22.59 & -26.80 \\
\hline NGC 5077 & E3 & $41.2[3 \mathrm{a}]$ & $\mathrm{y}[4 \mathrm{~h}]$ & 255 & $7.4_{-3.0}^{+4.7}[6 \mathrm{n}]$ & 12.38 & 8.216 & 0.210 & 0.018 & 0.04 & -0.02 & $\ldots$ & -20.91 & -24.94 \\
\hline NGC 5576 & E3 & 24.8 & $\mathrm{n}[4 \mathrm{~b}]$ & 171 & $1.6_{-0.4}^{+0.3}[6 \mathrm{o}]$ & 1.85 & .827 & 0.136 & 0.012 & 0.02 & -0.01 & $\ldots$ & -20.26 & -24.19 \\
\hline NGC 5813 & E1 & 31.3 & $\mathrm{y}[4 \mathrm{~b}]$ & 239 & $6.8_{-0.7}^{+0.7}[6 \mathrm{e}]$ & 11.45 & 413 & 0.246 & 0.021 & 0.03 & -0.01 & $\ldots$ & -21.28 & -25.12 \\
\hline NGC 5845 & $\mathrm{E} 4$ & 25.2 & $\mathrm{n}[4 \mathrm{~h}]$ & 238 & $2.6_{-1.5}^{+0.4}[6 \mathrm{c}]$ & 13.50 & 9.112 & 0.230 & 0.020 & 0.02 & -0.01 & $\ldots$ & -18 & -22.95 \\
\hline NGC 5846 & E0 & 24.2 & $\mathrm{y}[4 \mathrm{i}]$ & 237 & $11_{-1}^{+1}[6 \mathrm{e}]$ & 11.05 & 6.949 & 0.237 & 0.020 & 0.02 & -0.01 & $\ldots$ & -21 & -25.02 \\
\hline NGC 6086 & $\mathrm{E} 3$ & $138.0[3 \mathrm{a}]$ & $y[4 j]$ & $318[6 \mathrm{p}]$ & $37_{-11}^{+18}[6 \mathrm{p}]$ & 13.79 & 9.973 & 0.162 & 0.014 & 0.13 & -0.05 & ... & -22.08 & -25.93 \\
\hline NGC 6251 & $\mathrm{E} 2$ & $104.6[3 \mathrm{a}]$ & $\mathrm{y} ?[6 \mathrm{q}]$ & 311 & $5.9_{-2.0}^{+2.0} \quad[6 \mathrm{q}]$ & $13.64[7 \mathrm{~b}]$ & 9.026 & 0.377 & 0.032 & 0.10 & -0.04 & $\ldots$ & -21.84 & -26.25 \\
\hline NGC 7052 & $\mathrm{E} 4$ & $66.4[3 \mathrm{a}]$ & $\mathrm{y}[4 \mathrm{k}]$ & 277 & $3.7_{-1.5}^{+2.6}[6 \mathrm{r}]$ & $13.40[7 \mathrm{~b}]$ & 8.574 & 0.522 & 0.044 & 0.06 & -0.03 & ... & -21.24 & -25.68 \\
\hline NGC 7768 & $\mathrm{E} 2$ & $112.8[6 \mathrm{~h}]$ & $\mathrm{y}[4 \mathrm{l}]$ & 257 [6h] & $13_{-4}^{+5}[6 \mathrm{~h}]$ & 13.24 & 9.335 & 0.167 & 0.014 & 0.11 & -0.05 & ... & -22 & -26.11 \\
\hline \multicolumn{15}{|c|}{45 Bulges } \\
\hline Cygnus A & $\mathrm{Sa} ?$ & $232.0[3 \mathrm{a}]$ & $\mathrm{y} ?$ & 270 & $25_{-7}^{+7}[6 \mathrm{~s}]$ & 17.04 & 10.276 & 1.644 & 0.140 & 0.17 & -0.07 & 0.13 & -20.80 & -25.81 \\
\hline IC 2560 & $\mathrm{SBb}$ & $40.7[3 a]$ & $\mathrm{n} ?[4 \mathrm{~m}]$ & $144[5 \mathrm{~d}]$ & $0.044_{-0.022}^{+0.044}[6 \mathrm{t}]$ & $12.53[7 \mathrm{~b}]$ & 8.694 & 0.410 & 0.035 & 0.02 & -0.02 & 0.20 & -19 & -22.78 \\
\hline NGC 224 & $\mathrm{Sb}$ & 0.74 & $\mathrm{n}[4 \mathrm{n}]$ & 170 & $1.4_{-0.3}^{+0.9}[6 \mathrm{u}]$ & 4.36 & 0.984 & 0.268 & 0.023 & 0.00 & -0.00 & 0.49 & -18.6 & -21.82 \\
\hline NGC 253 & $\mathrm{SBc}$ & $3.5[3 \mathrm{c}]$ & $\mathrm{n}[4 \mathrm{o}]$ & $109[5 \mathrm{e}]$ & $0.10_{-0.05}^{+0.10}[6 \mathrm{v}]$ & 8.04 & 3.772 & 0.081 & 0.007 & 0.00 & -0.00 & 0.61 & -17.33 & -21.42 \\
\hline NGC 524 & S0 & 23.3 & $\mathrm{y}[4 \mathrm{e}]$ & 253 & $8.3_{-1.3}^{+2.7}[6 \mathrm{w}]$ & 11.30 & 7.163 & 0.356 & 0.030 & 0.03 & -0.02 & 0.01 & -20.15 & -23.61 \\
\hline NGC 1023 & SB0 & 11.1 & $\mathrm{n}[4 \mathrm{~g}]$ & 204 & $0.42_{-0.04}^{+0.04}[6 \mathrm{x}]$ & 10.35 & 6.238 & 0.262 & 0.022 & 0.01 & -0.00 & 0.47 & -19.8 & -23.01 \\
\hline NGC 1068 & $\mathrm{SBb}$ & $15.2[3 \mathrm{a}]$ & $\mathrm{n}[4 \mathrm{p}]$ & $165[5 f]$ & $0.084_{-0.003}^{+0.003}[6 \mathrm{y}]$ & 9.61 & 5.788 & 0.145 & 0.012 & 0.01 & -0.01 & 0.07 & -19.45 & -23.47 \\
\hline NGC 1194 & So & $53.9[3 \mathrm{a}]$ & $\mathrm{n} ?$ & $148[5 \mathrm{~g}]$ & $0.66_{-0.03}^{+0.03}[6 z]$ & 13.83 & 9.758 & 0.330 & 0.028 & 0.05 & -0.03 & 0.25 & -19.58 & -22.91 \\
\hline NGC 1300 & $\mathrm{SBbc}$ & 20.7 [3a] & $\mathrm{n}[4 \mathrm{q}]$ & 229 & $0.73_{-0.35}^{+0.69}[6 \mathrm{aa}]$ & 11.11 & 7.564 & 0.130 & 0.011 & 0.01 & -0.01 & 0.18 & -18.05 & -21.91 \\
\hline NGC 1316 & SB0 & $18.6[3 \mathrm{~d}]$ & $?$ & 226 & $1.5_{-0.8}^{+0.75}[6 \mathrm{ab}]$ & 9.42 & 5.587 & 0.090 & 0.008 & 0.02 & -0.02 & 0.15 & -21.33 & -24.68 \\
\hline NGC 1332 & So & 22.3 & $\mathrm{y} ?$ & 320 & $14.5_{-2}^{+2}[6 \mathrm{ac}]$ & 11.25 & 7.052 & 0.141 & 0.012 & 0.02 & -0.01 & 0.51 & -20.37 & -23.74 \\
\hline NGC 2273 & $\mathrm{SBa}$ & $28.5[3 \mathrm{a}]$ & $\mathrm{n}[4 \mathrm{r}]$ & $145[5 \mathrm{~g}]$ & $0.083_{-0.004}^{+0.004}[6 \mathrm{z}]$ & 12.55 & 8.480 & 0.305 & 0.026 & 0.02 & -0.01 & 0.12 & -19.33 & -22.66 \\
\hline NGC 2549 & SB0 [2a] & 12.3 & $\mathrm{n}[4 \mathrm{q}]$ & 144 & $0.14_{-0.13}^{+0.02}[6 \mathrm{w}]$ & 12.19 & 8.046 & 0.282 & 0.024 & 0.01 & -0.01 & 0.48 & -18.24 & -21.44 \\
\hline NGC 2778 & $\mathrm{SB0}[2 \mathrm{~b}]$ & 22.3 & $\mathrm{n}[4 \mathrm{~d}]$ & 162 & $0.15_{-0.1}^{+0.09}[6 \mathrm{c}]$ & 13.35 & 9.514 & 0.090 & 0.008 & 0.02 & -0.02 & 0.14 & -17.79 & -21.15 \\
\hline NGC 2787 & SB0 & 7.3 & $\mathrm{n}[4 \mathrm{~s}]$ & 210 & $0.40_{-0.05}^{+0.04}[6 \mathrm{ad}]$ & 11.82 & 7.263 & 0.565 & 0.048 & 0.01 & -0.00 & 0.19 & -17.41 & -20.98 \\
\hline NGC 2960 & $\mathrm{Sa} ?$ & $81.0[3 \mathrm{e}]$ & $\mathrm{n} ?$ & $166[5 \mathrm{~g}]$ & $0.117_{-0.005}^{+0.005}[6 \mathrm{z}]$ & $13.29[7 \mathrm{~b}]$ & 9.783 & 0.193 & 0.016 & 0.05 & -0.03 & 0.16 & -20.79 & -23.69 \\
\hline NGC 3031 & Sab & 3.8 & $\mathrm{n}[4 \mathrm{t}]$ & 162 & $0.74_{-0.11}^{+0.21}[6 \mathrm{ae}]$ & 7.89 & 3.831 & 0.346 & 0.029 & 0.00 & -0.00 & 0.28 & -19.59 & -22.56 \\
\hline NGC 3115 & So & 9.4 & $\mathrm{n}[4 \mathrm{a}]$ & 252 & $8.8_{-2.7}^{+10.0}[6 \mathrm{af}]$ & 9.87 & 5.883 & 0.205 & 0.017 & 0.01 & -0.01 & 0.47 & -19.88 & -23.00 \\
\hline NGC 3227 & $\mathrm{SBa}$ & $20.3[3 a]$ & $\mathrm{n}[4 \mathrm{e}]$ & 133 & $0.14_{-0.06}^{+0.10}[6 \mathrm{ag}]$ & 11.10 & 7.639 & 0.098 & 0.008 & 0.01 & -0.01 & 0.17 & -19.87 & -22.75 \\
\hline NGC 3245 & So & 20.3 & $\mathrm{n}[4 \mathrm{e}]$ & 210 & $2.0_{-0.5}^{+0.5}[6 \mathrm{ah}]$ & 11.70 & 7.862 & 0.108 & 0.009 & 0.02 & -0.01 & 0.26 & -19.37 & -22.61 \\
\hline NGC 3368 & SBab & 10.1 & $\mathrm{n}[4 \mathrm{u}]$ & 128 & $0.073_{-0.015}^{+0.015}$ [6ai] & 10.11 & 6.320 & 0.109 & 0.009 & 0.01 & -0.01 & 0.16 & -19.14 & -22.17 \\
\hline NGC 3384 & SB0 & 11.3 & $\mathrm{n}[4 \mathrm{a}]$ & 148 & $0.17_{-0.02}^{+0.01}[6 \mathrm{c}]$ & 10.85 & 6.750 & 0.115 & 0.010 & 0.01 & -0.01 & 0.34 & -19.05 & -22.47 \\
\hline NGC 3393 & $\mathrm{SBa}$ & $55.2[3 \mathrm{a}]$ & $\mathrm{n}[4 \mathrm{~m}]$ & 197 & $0.34_{-0.02}^{+0.02}[6 \mathrm{aj}]$ & $13.09[7 \mathrm{~b}]$ & 9.059 & 0.325 & 0.028 & 0.04 & -0.02 & 0.04 & -20.21 & -23.56 \\
\hline NGC 3414 & S0 & 24.5 & $\mathrm{n}[4 \mathrm{e}]$ & 237 & $2.4_{-0.3}^{+0.3}[6 \mathrm{e}]$ & 11.96 & 7.981 & 0.106 & 0.009 & 0.02 & -0.01 & 0.14 & -19.40 & -22.87 \\
\hline
\end{tabular}


TABLE 1

cont.

\begin{tabular}{|c|c|c|c|c|c|c|c|c|c|c|c|c|c|c|}
\hline $\begin{array}{c}\text { Galaxy } \\
\text { (1) }\end{array}$ & Type & $\begin{array}{c}\text { Dist } \\
{[\mathrm{Mpc}]} \\
(3)\end{array}$ & (4) & $\begin{array}{c}\sigma \\
\mathrm{km} \mathrm{s}_{(5)}^{-1}\end{array}$ & $\begin{array}{c}M_{\mathrm{bh}} \\
{\left[10^{8} M_{\odot}\right]} \\
(6)\end{array}$ & $\begin{array}{c}B_{T} \\
{[\mathrm{mag}]} \\
(7)\end{array}$ & $\begin{array}{c}K_{s} \\
{[\mathrm{mag}]} \\
(8)\end{array}$ & $\begin{array}{c}\mathrm{A}_{B} \\
{[\mathrm{mag}]} \\
(9)\end{array}$ & $\begin{array}{c}\mathrm{A}_{K} \\
{[\mathrm{mag}]} \\
(10)\end{array}$ & $\begin{array}{c}\mathrm{K}_{B} \\
{[\mathrm{mag}]} \\
(11)\end{array}$ & $\begin{array}{c}\mathrm{K}_{K_{s}} \\
{[\mathrm{mag}]} \\
(12)\end{array}$ & $\begin{array}{l}\mathrm{R}_{25} \\
(13)\end{array}$ & $\begin{array}{c}\mathrm{M}_{B} \\
{[\mathrm{mag}]} \\
(14)\end{array}$ & $\begin{array}{c}\mathrm{M}_{K_{s}} \\
{[\mathrm{mag}]} \\
(15)\end{array}$ \\
\hline NGC 3489 & SB0 & 11.7 & $\mathrm{n}[4 \mathrm{q}]$ & 105 & $0.058_{-0.008}^{+0.008}[6 \mathrm{ai}]$ & 11.12 & 7.370 & 0.072 & 0.006 & 0.01 & -0.01 & 0.24 & -18.69 & -21.88 \\
\hline NGC 3585 & S0 & 19.5 & $\mathrm{n}[4 \mathrm{q}]$ & 206 & $1_{-0.6}^{+1.4}[6 \mathrm{o}]$ & 10.88 & 6.703 & 0.276 & 0.023 & 0.02 & & 0.26 & & -23.69 \\
\hline NGC 3607 & S0 & 22.2 & $\mathrm{n}[4 \mathrm{e}]$ & 224 & $3_{-0.5}^{+0.5}[6 \mathrm{o}]$ & 10.82 & 6.994 & 0.090 & 0.008 & 0.01 & -0.01 & 0.30 & & -23.68 \\
\hline NGC 3998 & So & 13.7 & $\mathrm{y} ?[4 \mathrm{v}]$ & 305 & $1_{-1.9}^{+2.0}[6 \mathrm{ak}]$ & 11.61 & 7.365 & 0.069 & 0.006 & 0.01 & -0.01 & 0.08 & -18 & -22.21 \\
\hline NGC 4026 & So & 13.2 & $\mathrm{n}[4 \mathrm{q}]$ & 178 & $8_{-0.3}^{+0.6}[6 o]$ & 11.67 & 7.584 & 0.095 & 0.008 & 0.01 & -0 & 0.61 & -1 & -22.11 \\
\hline NGC 4151 & SBab & $20.0[3 \mathrm{a}]$ & $\mathrm{n}[4 \mathrm{e}]$ & 156 & $5_{-0.07}^{+0.07}[6 \mathrm{al}]$ & 11.50 & 7.381 & 0.119 & 0.010 & 0.01 & -0.01 & 0.15 & -19.24 & -22.60 \\
\hline NGC 4258 & $\mathrm{SBbc}$ & $7.2[3 \mathrm{f}]$ & $\mathrm{n}[4 \mathrm{w}]$ & 134 & $39_{-0.01}^{+0.01}[6 \mathrm{am}]$ & 9.10 & 5.464 & 0.069 & 0.006 & 0.00 & -0 . & 0.41 & & -21.76 \\
\hline NGC 4342 & So & $23.0[3 \mathrm{~g}]$ & $\mathrm{n}[4 \mathrm{x}]$ & 253 & $5_{-1.5}^{+2.3}[6 \mathrm{an}]$ & 13.41 & 9.023 & 0.088 & 0.008 & 0.01 & -0 & 0.33 & & -21.73 \\
\hline NGC 4388 & $\mathrm{Sb}$ & $17.0[3 \mathrm{~b}]$ & $\mathrm{n} ?[4 \mathrm{y}]$ & $107[5 \mathrm{~g}]$ & $75_{-0.002}^{+0.002}[6 z]$ & 11.76 & 8.004 & 0.143 & 0.012 & 0.02 & -0.02 & 0.64 & -20.07 & -23.65 \\
\hline NGC 4459 & S0 & 15.7 & $\mathrm{n}[4 \mathrm{f}]$ & 178 & $68_{-0.13}^{+0.13}[6 \mathrm{ad}]$ & 11.32 & 7.152 & 0.199 & 0.017 & 0.01 & -0 & 0.12 & & -22.73 \\
\hline NGC 4564 & So $[2 \mathrm{c}]$ & 14.6 & $\mathrm{n}[4 \mathrm{f}]$ & 157 & $.60_{-0.09}^{+0.03}[6 \mathrm{c}]$ & 12.05 & 7.937 & 0.151 & 0.013 & 0.01 & -0 & 0.38 & & -21.87 \\
\hline NGC 4594 & $\mathrm{Sa}$ & 9.5 & $\mathrm{y}[6 \mathrm{ao}]$ & 297 [6ao] & $4_{-0.4}^{+0.4}[6 \mathrm{ao}]$ & 8.98 & 4.962 & 0.221 & 0.019 & 0.01 & -0.01 & 0.39 & -2 & -23.86 \\
\hline NGC 4596 & SB0 & $17.0[3 \mathrm{~b}]$ & $\mathrm{n}[4 \mathrm{z}]$ & 149 & $.79_{-0.33}^{+0.38}[6 \mathrm{ad}]$ & 11.35 & 7.463 & 0.096 & 0.008 & 0.02 & -0 . & 0.13 & & -22.60 \\
\hline NGC 4736 & $a b$ & $4.4[3 \mathrm{i}]$ & $\mathrm{n} ?$ & 104 & $0.060_{-0.014}^{+0.014}[6 \mathrm{ap}]$ & 8.99 & 5.106 & 0.076 & 0.007 & 0.00 & -0.00 & 0.09 & & -21.55 \\
\hline NGC 4826 & $\mathrm{Sab}$ & 7.3 & $\mathrm{n} ?$ & 91 & $016_{-0.004}^{+0.004}[6 \mathrm{ap}]$ & 9.36 & 5.330 & 0.178 & 0.015 & 0.00 & -0.00 & 0.27 & -19.36 & -22.47 \\
\hline NGC 4945 & SBcd & $3.8[3 \mathrm{i}]$ & $\mathrm{n} ?[4 \mathrm{aa}]$ & 100 & $.014_{-0.007}^{+0.014}[6 \mathrm{aq}]$ & 9.30 & 4.483 & 0.762 & 0.065 & 0.00 & -0 & 0.72 & & -20.60 \\
\hline NGC 5128 & So & $3.8[3 \mathrm{i}]$ & $\mathrm{n} ?[4 \mathrm{ab}]$ & 120 & $45_{-0.10}^{+0.17}[6 \mathrm{ar}]$ & 7.84 & 3.942 & 0.496 & 0.042 & 0.01 & -0.00 & 0.11 & -19.84 & -22.87 \\
\hline NGC 6264 & $\mathrm{Sa} ?$ & $146.3[3 \mathrm{a}]$ & $\mathrm{n} ?$ & $159[5 \mathrm{~g}]$ & $0.305_{-0.004}^{+0.004}[6 \mathrm{z}]$ & $15.42[7 \mathrm{~b}]$ & 11.407 & 0.280 & 0.024 & 0.10 & -0.07 & 0.15 & -20 & -23.47 \\
\hline NGC 6323 & $\mathrm{SBab}[2 \mathrm{~d}]$ & $112.4[3 \mathrm{a}]$ & $\mathrm{n} ?$ & $159[5 \mathrm{~g}]$ & $0.10_{-0.001}^{+0.001}[6 \mathrm{z}]$ & $14.85[7 \mathrm{~b}]$ & 10.530 & 0.074 & 0.006 & 0.06 & -0 . & 0.48 & & -23.44 \\
\hline NGC 7582 & SBab & $22.0[3 \mathrm{a}]$ & $\mathrm{n}[4 \mathrm{ac}]$ & 156 & $0.55_{-0.19}^{+0.26}[6 \mathrm{as}]$ & 11.37 & 7.316 & 0.061 & 0.005 & 0.01 & -0.01 & 0.38 & $-1 \mathrm{c}$ & -22.94 \\
\hline UGC 3789 & SBab & $48.4[3 \mathrm{a}]$ & $\mathrm{n} ?$ & $107[5 \mathrm{~g}]$ & $0.108_{-0.005}^{+0.005}[6 \mathrm{z}]$ & $13.30[7 \mathrm{~b}]$ & 9.510 & 0.280 & 0.024 & 0.03 & -0.04 & 0.06 & -19.48 & -22.46 \\
\hline NGC 3079 & $\mathrm{SBc}$ & 20.7 [3a] & $\mathrm{n} ?[4 \mathrm{ad}]$ & $60-150[5 \mathrm{~h}]$ & $0.024_{-0.012}^{+0.024}[6 \mathrm{at}]$ & 11.54 & 7.262 & 0.049 & 0.004 & 0.01 & -0.00 & 0.74 & -17.80 & -21.86 \\
\hline Milky Way & $\mathrm{SBbc}$ & 0.008 [6au] & $\mathrm{n}[4 \mathrm{ae}]$ & $100[5 \mathrm{i}]$ & $0.043_{-0.004}^{+0.004}[6 \mathrm{au}]$ & $\ldots$ & $\ldots$ & $\cdots$ & $\cdots$ & $\ldots$ & $\ldots$ & $\ldots$ & $\ldots$ & $\ldots$ \\
\hline M32 & $\mathrm{E} ?[2 \mathrm{e}]$ & 0.79 & $\mathrm{n}[4 \mathrm{ae}]$ & $55[5 \mathrm{j}]$ & $0.024_{-0.005}^{+0.005}[6 \mathrm{av}]$ & 9.03 & 5.095 & 0.268 & 0.023 & 0.00 & -0.00 & 0.13 & -15.73 & -19.42 \\
\hline
\end{tabular}

Note. - Column 1: Galaxy name. Column 2: Basic morphological type, primarily from NED ${ }^{a}$. Column 3: Distance, primarily from Tonry et al. (2001) and corrected according to Blakeslee et al. (2002). Column 4: Presence of a partially depleted core. The addition of a question mark is used to indicate that this classification has come from the velocity dispersion criteria mentioned in the text. Column 5 : Velocity dispersion primarily from HyperLeda ${ }^{b}$ (Paturel et al. 2003). Column 6: Black hole mass, adjusted to the distances in column 3. Column 7: $B_{T}$ is the total (observed) magnitude in the $B$ system from the RC3, unless otherwise noted. Column 8: $K_{s}$ is the total 2MASS $K_{s}$-band magnitude. Columns $9 \& 10$ : The $B$ and $K$-band Galactic extinction from Schlegel et al. (1998). Columns $11 \& 12$ : The $B$ and $K$-band K-correction. Column $13: \mathrm{R}_{25}$, taken from the RC3, is the (base 10) logarithm of the major-to-minor $(a / b)$ isophotal axis ratio at $\mu_{B}=25 \mathrm{mag} \operatorname{arcsec}^{-2}$. Columns $14 \& 15$ : Corrected, absolute, $B$ - and $K$-band spheroid magnitude. References: $2 \mathrm{a}=$ Krajnović et al. (2009); 2b = Rix et al. (1999); 2c = Trujillo et al. (2004); 2d = Jarrett et al. (2000) 2MASS image; 2e = Graham (2002); 3a = NED (Virgo + GA + Shapley)-corrected Hubble flow distance; 3b = Jerjen et al. (2004); 3c = Rekola et al. (2005); 3d = Madore et al. (1999); 3e = Violette Impellizzeri et al. (2012); 3f = Herrnstein et al. (1999); 3g = Mei et al. (2007), Blakeslee et al. (2009), Blom et al. (2012), Bogdán et al. (2012b); 3h = Herrmann et al. (2008); 3i = Karachentsev et al. (2007); 4a = Ravindranath et al. (2001); 4b = Dullo \& Graham (2012); 4c = Lauer et al. (2005); 4d = Rest et al. (2001); 4e = Richings et al. (2011); $4 \mathrm{f}=$ Ferrarese et al. (2006); $4 \mathrm{~g}=$ Faber et al. (1997); $4 \mathrm{~h}=$ Trujillo et al. (2004); 4i = Forbes, Brodie, \& Huchra (1997); 4j = Laine et al. (2003); 4k = Quillen et al. (2000); $4 \mathrm{l}=$ Grillmair et al. (1994); 4m = Muñoz Marín et al. (2007); 4n = Corbin, O’Neil, \& Rieke (2001); 4o = Kornei \& McCrady (2009); 4p = Macchetto et al. (1994); 4q = Graham (2012b); 4r = Malkan, Gorjian \& Tam (1998); 4s = Erwin et al. (2003); $4 \mathrm{t}=$ Fisher \& Drory (2008); $4 \mathrm{u}=$ Nowak et al. (2010); 4v = González Delgado et al. (2008); 4w = Pastorini et al. (2007); 4x = van den Bosch, Jaffe, \& van der Marel (1998); 4y = Pogge \& Martini (2002); 4z = Gerssen, Kuijken, \& Merrifield (2002); 4aa = Marconi et al. (2000); $4 \mathrm{ab}=$ Radomski et al. (2008); 4ac = Wold \& Galliano (2006); 4ad = Cecil et al. (2001); 4ae = Graham \& Spitler (2009); 5a = Dalla Bontà et al. (2009); 5b = Smith et al. (2000); 5c = De Francesco et al. (2006); 5d = Cid Fernandes et al. (2004); 5e = Oliva et al. (1995); $5 \mathrm{f}=$ Nelson \& Whittle (1995); $5 \mathrm{~g}=$ Greene et al. (2010); $5 \mathrm{~h}=$ Shaw, Wilkinson, \& Carter (1993); 5i = Merritt \& Ferrarese (2001a); 5j = Lucey et al. (1997), Chilingarian (2012, in prep.); 6a = Dalla Bontà et al. (2009); 6b = Cappellari et al. (2002), stellar dynamical measurement; 6c = Gebhardt et al. (2003), Gültekin et al. (2009b); 6d = Houghton et al. (2006), Gebhardt et al. (2007); 6e $=$ Preliminary values determined by $\mathrm{Hu}$ (2008) from Conf. Proc. figures of Cappellari et al. (2008); $6 \mathrm{f}=\mathrm{Copin}$ et al. (2004); 6g = van den Bosch \& de Zeeuw (2010); $6 \mathrm{~h}=$ McConnell et al. (2012); $6 \mathrm{i}=$ Ferrarese et al. (1996); $6 \mathrm{j}=$ Walsh et al. (2010); 6k = Gebhardt et al. (2011); $6 \mathrm{l}=$ Nowak et al. (2007); 6m = Shen \& Gebhardt (2009); 6n = De Francesco et al. (2008); 6o = Gültekin et al. (2009a); 6p = McConnell et al. (2011b); 6q = Ferrarese \& Ford (1999); 6r = van der Marel \& van den Bosch (1998); 6s = Tadhunter et al. (2003); 6t = Ishihara et al. (2001), Nakai et al. (1998); 6u = Bacon et al. (2001), Bender et al. (2005); 6v = Rodríguez-Rico et al. (2006), a factor of 2 uncertainty has been assigned here; $6 \mathrm{w}=$ Krajnović et al. (2009); 6x = Bower et al. (2001); 6y = Lodato \& Bertin (2003); 6z $=$ Kuo et al. (2011); 6aa = Atkinson et al. (2005); 6ab = Nowak et al. (2008); 6ac = Rusli et al. (2011); 6ad = Sarzi et al. (2001); 6ae = Devereux et al. (2003); 6af = Emsellem et al. (1999); $6 \mathrm{ag}=$ Davies et al. (2006), Hicks \& Malkan (2008); 6ah = Barth et al. (2001); 6ai $=$ Nowak et al. (2010); 6aj = Kondratko et al. (2008); 6ak = Walsh et al. (2012); 6al = Onken et al. (2007), Hicks \& Malkan (2008); $6 \mathrm{am}=$ Herrnstein et al. (1999); 6an = Cretton \& van den Bosch (1999), Valluri et al. (2004); 6ao = Jardel et al. (2011), NGC 4594 is a core-Sérsic galaxy with an AGN; 6ap = Kormendy et al. (2011), priv. value from K.Gebhardt; 6aq = Greenhill et al. (1997); 6ar = Neumayer (2010); 6as = Wold et al. (2006); 6at = Trotter et al. (1998), Yamauchi et al. (2004), Kondratko et al. (2005); 6au = Gillessen et al. (2009); $6 \mathrm{av}=$ Verolme et al. (2002); $7 \mathrm{a}=B_{T}$ (Johnson), Gavazzi et al. (2005); $7 \mathrm{~b}=$ The RC3's $m_{B}$ value (which they reduced to the $B_{T}$ system); $8 \mathrm{a}=$ Cappellari et al. (2006).

${ }^{a}$ http://nedwww.ipac.caltech.edu

${ }^{b}$ http://leda.univ-lyon1.fr 
TABLE 2

AVERAGE DUST-CORRECTED (INTRINSIC) BULGE-TO-DISK FLUX RATIOS

\begin{tabular}{lcc}
\hline \multicolumn{3}{c}{ Galaxy $<\log (B / D)><\log (B / D)>$} \\
Type & $B$-band & K-band \\
\hline S0/S0a & -0.29 & -0.31 \\
Sa & -0.29 & -0.34 \\
Sab & -0.39 & -0.54 \\
Sb & -0.87 & -0.60 \\
Sbc & -1.13 & -0.82 \\
Sc & -1.28 & -1.06 \\
Scd & -1.55 & -1.23 \\
Adapted from & Graham \& Worley $(2008)$.
\end{tabular}

assign the core type. This meant that for seven galaxies with $\sigma>270 \mathrm{~km} \mathrm{~s}^{-1}$, all but one of which actually have $\sigma>300 \mathrm{~km} \mathrm{~s}^{-1}$, we classified them as coreSérsic galaxies. For 12 galaxies with $\sigma \leq 166 \mathrm{~km} \mathrm{~s}^{-1}$ we have classified them as Sérsic galaxies. This approach relates to the trend in which low-luminosity spheroids, often found in disk galaxies, do not possess partially depleted cores while luminous spheroids do (e.g. Nieto et al. 1991; Ferrarese et al. 1994; Faber et al. 1997). For the remaining galaxies, their core type was determined from high-resolution images (see column 4 of Table 1).

While Faber et al. (1997) reported that the peculiar galaxy NGC 1316 (Fornax A) has a partially-depleted core, we feel that a proper bulge/disk/bar decomposition - which is beyond the scope of this paper - might be required for this barred S0 galaxy. This may reveal a bulge with a small Sérsic index and a shallow inner profile slope, rather than an actual depleted core. Although NGC 1316 is a merger remnant (Deshmukh et al. 2012, and references therein) - which may support the presence of a depleted core if it had been a dry merger event - the presence of much dust and gas, plus the existence of a bar, is indicative of a minor, wet merger. We note that no other barred galaxy in our sample has a core which is partially depleted of stars, and to be safe we felt it best to exclude this galaxy from our final analysis until a more detailed investigation of its stellar distribution is performed.

\section{ANALYSIS AND RESULTS}

Our initial investigation of the $M_{\mathrm{bh}}-\sigma$ diagram uses all 75 galaxies (including NGC 1316) plus M32 and the Milky Way's bulge to give a total sample of 77 galaxies. It is apparent that there is something unusual with the two ultramassive black holes reported for NGC 3842 and NGC 4889 by McConnell et al. (2011a). Figure1 1 reveals that they may be are outliers biasing the $M_{\mathrm{bh}}-\sigma$ relation defined by the remaining data. Performing a symmetrical linear regression on the core-Sérsic galaxies, including NGC 3842 and NGC 4889, yields a slope of roughly $7.0 \pm 1$ (see Table 31). Uncertain as to whether there is an error in the black hole masses for NGC 3842 and NGC 4889, or perhaps with their velocity dispersion measurements, or if instead these values are fine and these galaxies are representative (of a possibly new class) of galaxies which deviate from the $M_{\mathrm{bh}}-\sigma$ relation as we know it, in the following section we have elected to repeat the $M_{\mathrm{bh}}-\sigma$ regression analysis without these two galaxies. If these galaxies are outliers, then this additional approach provides a more robust statistical analysis of the $M_{\mathrm{bh}}-\sigma$ re- lation. To avoid issues of inconsistent samples, these two galaxies are also excluded from the ensuing $M_{\mathrm{bh}}-L$ diagram, although doing so has no significant effect on our $M_{\mathrm{bh}}-L$ relation.

Although NGC 1316 is not an outlier from the $M_{\mathrm{bh}}-\sigma$ diagram (Figure 1), we do additionally exclude it from what follows due to the previously mentioned uncertainty associated with its core type. If NGC 1316 is confirmed to possess a partially-depleted core, something associated with dry merger events, then it will be the only such example in a barred galaxy (with bars of course associated with secular evolution rather than dry mergers). Until a three-component Sérsic-bulge plus exponential-disk plus bar model has been fit to this galaxy, it is premature to claim from a fitted single-component, core-Sérsic model that this galaxy has a depleted core.

Our cautious exclusion of these three galaxies (NGC 1316, NGC 3842 and NGC 4889), leaves us with our final sample of $(75-3=) 72$ galaxies, comprised of 24 core-Sérsic galaxies and 48 Sérsic galaxies.

\subsection{The $M_{\mathrm{bh}}-\sigma$ diagram}

In Figure 2a we have performed a symmetrical linear regression for the 24 core-Sérsic galaxies in the $M_{\mathrm{bh}}-\sigma$ diagram. This was achieved using the bisector regression analysis in the BCES routine from Akritas \& Bershady (1996). The results are provided in Table 3 Of note is that the symmetrical $M_{\mathrm{bh}}-\sigma$ relation has a slope consistent with a value of 5 (Ferrarese \& Merritt 2000; Hu 2008; Graham et al. 2011).

We have additionally performed the same symmetrical regression for the Sérsic sample (see Table 3). However, due to each supermassive black hole's limited sphere-ofinfluence, $r_{\mathrm{infl}} \sim G M_{\mathrm{bh}} / \sigma^{2}$ (Merritt \& Ferrarese 2001b), there is an associated galaxy distance limit to which we can spatially resolve the black hole's dynamical influence on its surrounding gas and stars. Shown in Figure 2a is the limit to which one can detect black holes at a distance of $1.3 \mathrm{Mpc}$ when the spatial resolution is $0^{\prime \prime} .1$ (i.e. roughly that achievable with the Hubble Space Telescope and ground-based adaptive-optics-assisted observations). For galaxies further away, this limit moves vertically in the diagram to higher black hole masses. Attempts to occupy the lower right of Figure 2a may need to wait for the next generation of telescopes with greater spatial resolution, and as such we currently have a selection limit, a kind of floor, in this diagram (Batcheldor 2010; Schulze \& Wisotzki 2011). Galaxies can exist below this floor, but they are at distances such that we can not resolve their black hole's sphere-of-influence. To avoid this sample selection bias on the samples which extend into the lower portion of the diagram, we have additionally performed an ordinary least squares (OLS) regression of the abscissa (i.e., the horizontal $X$ value) on the ordinate (i.e., the vertical $Y$ value) for the reasons expounded in Lynden-Bell et al. (1988, their Figure 10).

Given that the 27 non-barred Sérsic galaxies define an $M_{\mathrm{bh}}-\sigma$ relation which is consistent with that defined by the 24 core-Sérsic galaxies (see Table 3), they have been combined to produce a single non-barred $M_{\mathrm{bh}}-\sigma$ relation. These 51 galaxies yield a slope of $5.53 \pm 0.34$, consistent with the value of $5.32 \pm 0.49$ reported by Graham et al. (2011) using a sample of 44 non-barred galaxies. Barth, Greene, \& Ho (2005, their figure 2) offers further support 


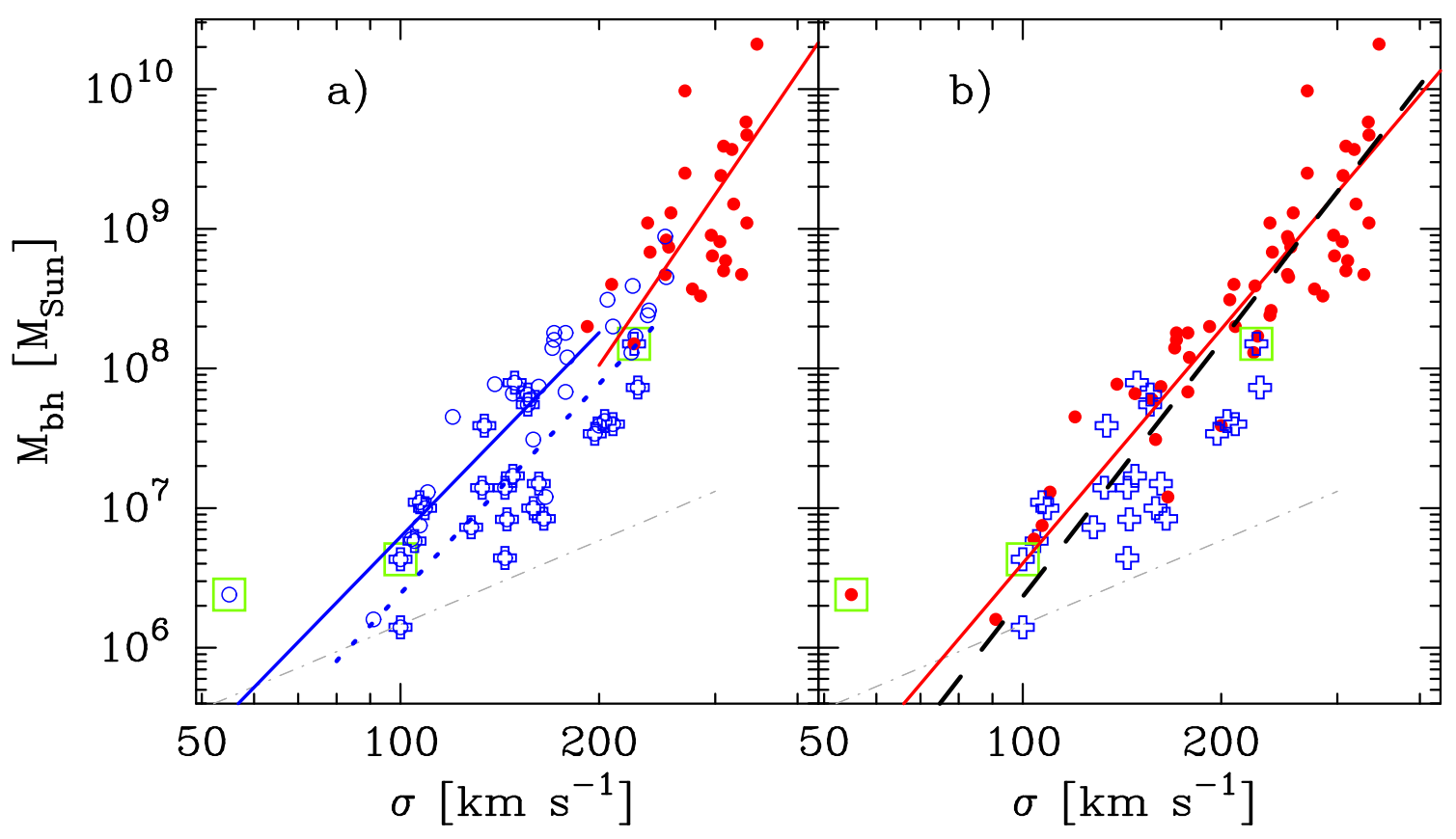

FIG. 1. - Black hole mass versus central velocity dispersion. The faint dot-dashed gray line corresponds to a black hole's sphere-ofinfluence of $0^{\prime \prime} .1$ at a distance of $1.3 \mathrm{Mpc}$. With such a limiting spatial resolution of $0^{\prime \prime} .1$, black holes residing below this line cannot be reliably detected. Panel a) Red dots represent the core-Sérsic galaxies, open blue circles represent the Sérsic galaxies, while the open crosses designate those which are barred. All 77 galaxies shown here have been included in the analysis of this Figure. The upper solid red line corresponds to the symmetrical regression for the 27 core-Sérsic galaxies, while (due to the sample selection limit) the dotted and solid blue lines correspond to the 'ordinary least squares' OLS $\left(\sigma \mid M_{\mathrm{bh}}\right)$ regression for the barred and non-barred Sérsic galaxies (see Table 31). Panel b) Red dots represent the non-barred galaxies while the open blue crosses designate those which are barred. The solid red line is a fit to the non-barred galaxies, while the dashed black line is a fit to all galaxies. M32, the Milky Way and Fornax A (NGC 1316) have been marked with a green square, they are not included in Figure 2 for the reasons mentioned in Section 2 The Sérsic galaxy with the highest black hole mass is NGC 3115 (see section 2.4).

for this linear relation after reducing their active galactic nuclei (AGN) black hole mass estimates by a factor of $\sim 2$ due to a revision in the virial $f$-factor used to determine black hole masses in AGN (Onken et al. 2004; Graham et al. 2011). While Graham et al. (2011) discussed why a $5 \%$ uncertainty on the velocity dispersion may be optimistic, we note that using a $10 \%$ uncertainty on $\sigma$, rather than $5 \%$, results in the same slope to the $M_{\mathrm{bh}}-\sigma$ relation when using the $\operatorname{OLS}\left(\sigma \mid M_{\mathrm{bh}}\right)$ regression. Coupling the knowledge that $L \propto \sigma^{2}$ for the Sérsic spheroids, with the relation $M_{\mathrm{bh}} \propto \sigma^{5.5}$, one would expect to find $M_{\mathrm{bh}} \propto L^{2.75}$ for the Sérsic spheroids.

For a value of $\sigma=400 \mathrm{~km} \mathrm{~s}^{-1}$, the non-barred $M_{\mathrm{bh}}-\sigma$ relation yields a black hole mass of $7.7 \times 10^{9} M_{\odot}$. This is 3 times greater than the value of $2.6 \times 10^{9} M_{\odot}$ predicted by the elliptical galaxy $M_{\mathrm{bh}}-\sigma$ relation from Gültekin et al. (2009b), and better, although not fully, matches the expectations from Hlavacek-Larrondo et al. (2012) for black holes in brightest cluster galaxies if they reside on the "fundamental plane of black hole activity" (Merloni et al. 2003; Falcke et al. 2004).

As reported by Graham (2007b, 2008a,b) and $\mathrm{Hu}$ (2008), the barred galaxies are, on average, offset to lower black hole masses or higher velocity dispersions than the non-barred galaxies, with the latter scenario possibly expected from barred galaxy dynamics (e.g. Gadotti \& Kauffmann 2009; Graham et al. 2011; DeBuhr, Ma \& White 2012). Consistent with Graham et al. (2011), we find a mean vertical offset of 0.30 dex between the barred and non-barred galaxies in the $M_{\mathrm{bh}}-\sigma$ diagram, which corresponds to a factor of $\sim 2$ in black hole mass, when $\sigma=200 \mathrm{~km} \mathrm{~s}^{-1}$.
For those who may not know if their galaxy of interest is barred or not, using the full 72 galaxies and an $\operatorname{OLS}\left(M_{\mathrm{bh}} \mid \sigma\right)$, bisector, and $\operatorname{OLS}\left(\sigma \mid M_{\mathrm{bh}}\right)$ regression to construct the classical (all galaxy type) $M_{\mathrm{bh}}-\sigma$ relation gives slopes of $5.21 \pm 0.27,5.61 \pm 0.27$ and $6.08 \pm$ 0.31 , respectively. The associated intercepts are $8.14 \pm$ $0.04,8.14 \pm 0.05$ and $8.15 \pm 0.05$. These steeper relations are expected given the offset nature of the barred galaxies.

\subsection{The $M_{\mathrm{bh}}-L$ diagram}

In Figures 2 $\mathrm{b}$ and $2 \mathrm{k}$ we have performed a symmetrical linear regression for the 24 core-Sérsic galaxies in the $M_{\mathrm{bh}}-L$ diagram. Expressing their spheroid magnitudes as luminosities, we find that $M_{\mathrm{bh}} \propto L_{K_{s}}^{1.10 \pm 0.20}$ and $M_{\mathrm{bh}} \propto L_{B}^{1.35 \pm 0.30}$, in reasonable agreement with the relation $M_{\mathrm{bh}} \propto L^{1.0}$ and with past analyses of predominantly bright galaxies ${ }^{10}$. We note that depending on the progenitor galaxy mass ratios - among the individual dry merger events which built one's core-Sérsic sample - the slope of the $M_{\mathrm{bh}}-L$ relation can be expected to deviate slightly from a value of 1 . Although, for galaxies built from a sufficient number of dry mergers, this should become a second order effect in terms of the overall evolutionary scenario in the $M_{\mathrm{bh}}-L$ diagram, for the reason described by Peng (2007) and Jahnke \& Maccio (2011).

The effective sample selection boundary shown in Figure $2 \mathrm{a}$ has been mapped into Figures $2 \mathrm{~b}$ and $2 \mathrm{c}$ by converting this line's velocity dispersion into the expected

10 The inclusion of NGC 3842 and NGC 4889 does not significantly change these results. 
The $M_{\mathrm{bh}}-L_{\text {spheroid }}$ relation

TABLE 3

BLACK HOLE SCALING RELATIONS

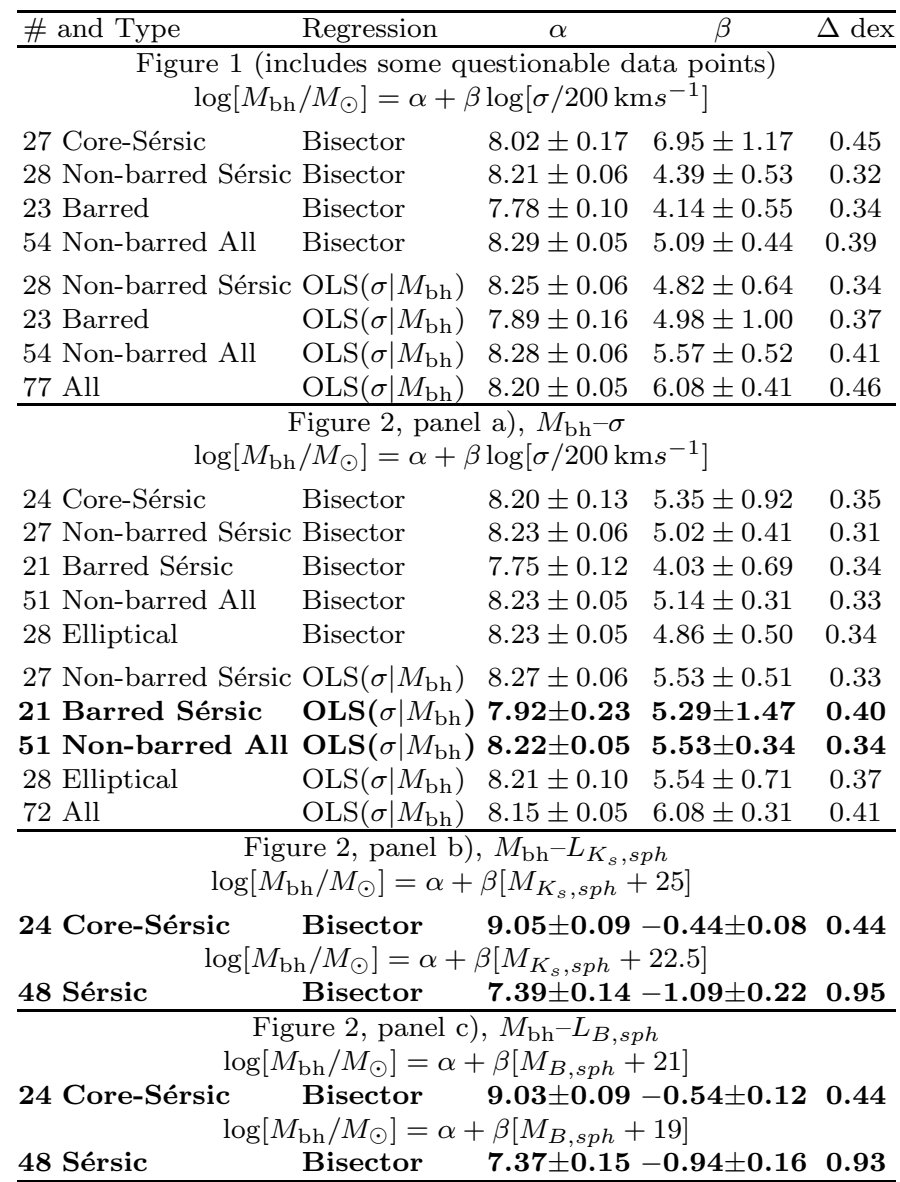

$M_{\mathrm{bh}}=$ black hole mass, $\sigma=$ host galaxy velocity dispersion, $M_{K_{s}, s p h}=$ dust-corrected, $2 \mathrm{MASS} K_{s}$-band spheroid magnitude, $M_{B, s p h}=$ dust-corrected, RC3 $B$-band spheroid magnitude. A symmetrical "bisector" regression was used, in addition to a non-symmetrical 'ordinary least squares' $\operatorname{OLS}\left(X \mid M_{\mathrm{bh}}\right)$ regression to compensate for the sample selection limit, or floor in the data, at the low-mass end (see Figure 2). The total rms scatter in the $\log M_{\mathrm{bh}}$ direction is given by $\Delta$, and roughly scales linearly with the slope (within each panel). The lines highlighted in bold show the preferred fits, see the text for details, while Table 4 provides revised $M_{\mathrm{bh}}-L_{K_{s}}$ relations according to Schombert \& Smith's (2012) correction of the 2MASS photometry.

magnitude according to the $L-\sigma$ relations defined by the Sérsic galaxies in these diagrams. The need for an $\operatorname{OLS}\left(L \mid M_{\mathrm{bh}}\right)$ regression on the Sérsic galaxies in the $M_{\mathrm{bh}}-L$ diagrams, rather than a symmetrical regression, is no longer as apparent because the 'floor' to the sample selection has significantly shifted/rotated.

It is not clear if the barred Sérsic galaxies are offset from the non-barred Sérsic galaxies in Figures $20 \mathrm{~b}$ and 2. and so we have therefore grouped them together in our analysis. The results of which, given in Table 3, are such that $M_{\mathrm{bh}} \propto L_{K_{s}}^{2.73 \pm 0.55}$ and $M_{\mathrm{bh}} \propto L_{B}^{2.35 \pm 0.40}$ for the Sérsic spheroids ${ }^{11}$. We note in passing that it is expected that the color-magnitude relation for Sérsic spheroids (e.g., Tremonti et al. 2004; Jiménez et al. 2011), as opposed to the flat color-magnitude relation for core-Sérsic galaxies (as discussed in Graham 2012c), will result in these two exponents not being equal to each other. However the current uncertainty on these two exponents does not allow us to detect this difference.

Finally, Schombert (2011) and Schombert \& Smith (2012, see their Appendix) have recently detailed a pho-

11 The inclusion of NGC 3079 does not change these results. tometry error in the 2MASS Extended Source Catalog (Jarrett et al. 2000), such that the total 2MASS magnitudes are, on average, $0.33 \mathrm{mag}$ too faint in the $J$-band. There is a similar offset in the $K_{s}$-band data (Schombert 2012 , priv. comm.) such that the $V-K_{s}$ colors are too blue by this amount. Therefore, for those using $K$ or $K_{s}$-band data not from the 2MASS catalog for the prediction of black hole masses, the normalizing values of 25 and 22.5 used in the $M_{\mathrm{bh}}-L_{K_{s}}$ equations shown in Table 3 should be increased by 0.33 to 25.33 and 22.83 . One can actually go a little further than this. Assigning a 1-sigma uncertainty of $0.33 / 2=0.165 \mathrm{mag}$ to this correction, that is, assuming that the scatter seen in Schombert \& Smith's figure 12 has a 1-sigma value of $0.33 / 2 \mathrm{mag}$, we can estimate the impact of this correction on our $K_{s^{-}}$ band $M_{\mathrm{bh}}-L$ relations. Because they were constructed using $N=24$ core-Sérsic galaxies and $N=48$ Sérsic galaxies, our 'normalizing' values of 25 and 22.5 mag have an additional uncertainty of $0.165 / \sqrt{24}$ and $0.165 / \sqrt{48}$ mag associated with them, which is a small but nonzero 0.034 and $0.024 \mathrm{mag}$, respectively. Given the slopes of our two $M_{\mathrm{bh}}-L_{K_{s}}$ relations, this is equivalent to increasing the uncertainty on their intercept from 0.09 to 
TABLE 4

Corrected $M_{\mathrm{bh}}-L_{K_{s}}$ SCALing RElations

\begin{tabular}{|c|c|c|c|}
\hline \# and Type & Regression & $\beta$ & $\Delta \operatorname{dex}$ \\
\hline \multicolumn{4}{|c|}{$\log \left[M_{\mathrm{bh}} / M_{\odot}\right]=\alpha+\beta\left[M_{K_{s}, s p h}+25.33\right]$} \\
\hline 24 Core-Sérsi & Bisector & $9.05 \pm 0.12-0.44 \pm 0.08$ & 0.44 \\
\hline \multicolumn{4}{|c|}{$\log \left[M_{\mathrm{bh}} / M_{\odot}\right]=\alpha+\beta\left[M_{K_{s}, s p h}+22.83\right]$} \\
\hline 48 Sérsic & Bisector & $7.39 \pm 0.16-1.09 \pm 0.22$ & 0.95 \\
\hline
\end{tabular}

0.12 and from 0.14 to 0.16 , respectively. The result of applying these corrections is shown in Table 4). While this is a rather minor adjustment to our equations, it remains true that those using the $2 \mathrm{MASS}$ catalog of $K_{s^{-}}$ band magnitudes for individual galaxies may have the true galaxy magnitude wrong by the extent shown by Schombert \& Smith (2012), which will thus affect their predicted black hole mass.

It may be worth providing one additional comment in regard to photometric errors. If the 2MASS magnitudes are shown to contain a systematic error such that a greater fraction of galaxy light at large radii is increasingly missed in the intrinsically brighter galaxies, i.e. those with extended light profiles, this will not explain the bend in the $M_{\mathrm{bh}}-L$ relation. Bulges in (often truncated) exponential disk are not significantly affected by this potential problem. The affect is greater in galaxies with higher Sérsic indices, specifically the core-Sérsic elliptical galaxies. Such a correction would therefore act to slightly reduce the slope of the core-Sérsic $M_{\mathrm{bh}}-L$ relation, thus increasing the apparent 'bend' in the $M_{\mathrm{bh}}-L$ diagram between the Sérsic and the core-Sérsic spheroids.

\subsection{Propagation of errors}

It is important to appreciate the uncertainties on the slope and intercept of the relations given in Tables 3 and 4. plus the associated intrinsic scatter $\epsilon$. For example, the maximum 1-sigma uncertainty on the predicted value of $M_{\mathrm{bh}}$ using the $M_{\mathrm{bh}}-L_{K_{s}}$ relation is acquired by assuming uncorrelated errors on the magnitude $M_{K_{s}}$ and the slope and intercept of the relation. Gaussian error propagation for the linear equation $y=(b \pm \delta b)(x \pm \delta x)+(a \pm \delta a)$, gives an error on $y$ equal to

$$
\begin{aligned}
\delta y & =\sqrt{(d y / d b)^{2}(\delta b)^{2}+(d y / d a)^{2}(\delta a)^{2}+(d y / d x)^{2}(\delta x)^{2}} \\
& =\sqrt{x^{2}(\delta b)^{2}+(\delta a)^{2}+b^{2}(\delta x)^{2}} .
\end{aligned}
$$

In the presence of intrinsic scatter $(\epsilon)$ in the $y$-direction, the uncertainty on $y$ is

$$
\delta y=\sqrt{x^{2}(\delta b)^{2}+(\delta a)^{2}+b^{2}(\delta x)^{2}+\epsilon^{2}} .
$$

For the Sérsic $M_{\mathrm{bh}}-L_{K_{s}}$ relation in Table 4, $x=$ $\left[M_{K_{s}, s p h}+22.83\right]$, and thus

$$
\begin{gathered}
\left(\delta \log M_{\mathrm{bh}} / M_{\odot}\right)^{2}=\left[M_{K_{s}, s p h}+22.83\right]^{2}(0.22)^{2}+(0.16)^{2} \\
+(-1.09)^{2}\left[\delta M_{K_{s}, s p h}\right]^{2}+\epsilon^{2},
\end{gathered}
$$

where $\delta M_{K_{s}, s p h}$ is the uncertainty associated with the spheroid's magnitude.

For the preferred non-barred $M_{\mathrm{bh}}-\sigma$ relation in Table 3. $x=\log \left(\sigma / 200 \mathrm{kms}^{-1}\right)$, so $d x / d \sigma=1 /[\ln (10) \sigma]$, and therefore

$$
\begin{gathered}
\left(\delta \log M_{\mathrm{bh}} / M_{\odot}\right)^{2}=\left[\log \left(\sigma / 200 \mathrm{~km} \mathrm{~s}^{-1}\right)\right]^{2}(0.34)^{2}+(0.05)^{2} \\
+[5.53 / \ln (10)]^{2}[\delta \sigma / \sigma]^{2}+(\epsilon)^{2} .
\end{gathered}
$$

Precise knowledge of the intrinsic scatter in the above equations is a difficult task because it requires an accurate knowledge of the measurement errors, which we do not claim to have. As such, the scatter in our current $M_{\mathrm{bh}}-L$ diagram probably cannot be used to help distinguish between competing formation scenarios, as has been cleverly proposed (e.g. Lahav et al. 2011; Shankar et al. 2012). The measurement errors should add in quadrature with the intrinsic scatter to give the total rms scatter $\Delta$. We can thus quickly proceed with a rough estimate of the intrinsic scatter. In terms of the vertical scatter about the $M_{\mathrm{bh}}-\sigma$ relation, we have seen from Graham et al. (2011, their Table 2 ) that $\epsilon \approx 3 \Delta / 4$. Here we shall use a rough value of 0.3 dex, which is consistent with the results in Gültekin et al. (2009b). For the $M_{\mathrm{bh}}-L$ relation, Graham (2007a, his Table 4) reported a similar value of around 0.3 dex for his predominantly luminous galaxy sample. Obviously studies which have attempted to measure this (see also Gültekin et al. 2009b) but failed to account for the bent nature of the $M_{\mathrm{bh}}-L$ relation will be in error at some level. We shall however use this value here for the core-Sérsic galaxies. For the fainter Sérsic galaxies, which follow an $M_{\mathrm{bh}}-L$ relation that may be some 2.5 times steeper, we use a value of 0.75 dex. This increase to the intrinsic scatter in the vertical direction is expected if the intrinsic scatter in the horizontal direction, i.e. for the magnitudes, is equal for both types of galaxy, i.e. core-Sérsic and Sérsic galaxies.

The above equations are used in Section 4.1 where we predict a number of black hole masses which are less than one million solar masses.

\subsection{Method of regression}

Park et al. (2012) explored how the BCES linear regression code from Akritas \& Bershady (1996) can produce a biased $M_{\mathrm{bh}}-\sigma$ relation if one's velocity dispersions have large measurement errors. They revealed that there is not a bias when these errors are less than $\sim 15 \%$, the uppermost value used in the literature. Park et al. (2012) additionally showed that the 'forward' and 'inverse' linear regression from the BCES code yield $M_{\mathrm{bh}}-\sigma$ relations which are consistent with those derived using the 'forward' and 'inverse' versions of the modified FITEXY expression from Tremaine et al. (2002). That there is agreement between these codes in the $M_{\mathrm{bh}}-\sigma$ diagram is not a new result (e.g. Novak et al. 2006; Graham \& Li 2009; Graham et al. 2011), but it may be a poorly appreciated point worthy of some explanation before using the modified FITEXY code to check on our BCES-derived $M_{\mathrm{bh}}-L$ relations.

It is known that a linear regression which minimizes the offset of data from a fitted line will typically yield a different result if one minimizes the offset in either the vertical or the horizontal direction (e.g. Feigelson \& Babu 1992). It is also known that minimization of the vertical offsets (the so-called 'forward' regression) yields a shallower slope than obtained through minimization of the horizontal offsets (the 'inverse' regression). Obviously neither of these are symmetrical regressions because there is a preferred variable, that is, they do not treat the $x$ and $y$ data equally and therefore they generate different fitted lines. The two lines from each of these non-symmetrical regressions can however be combined to produce a symmetrical regression. For example, 


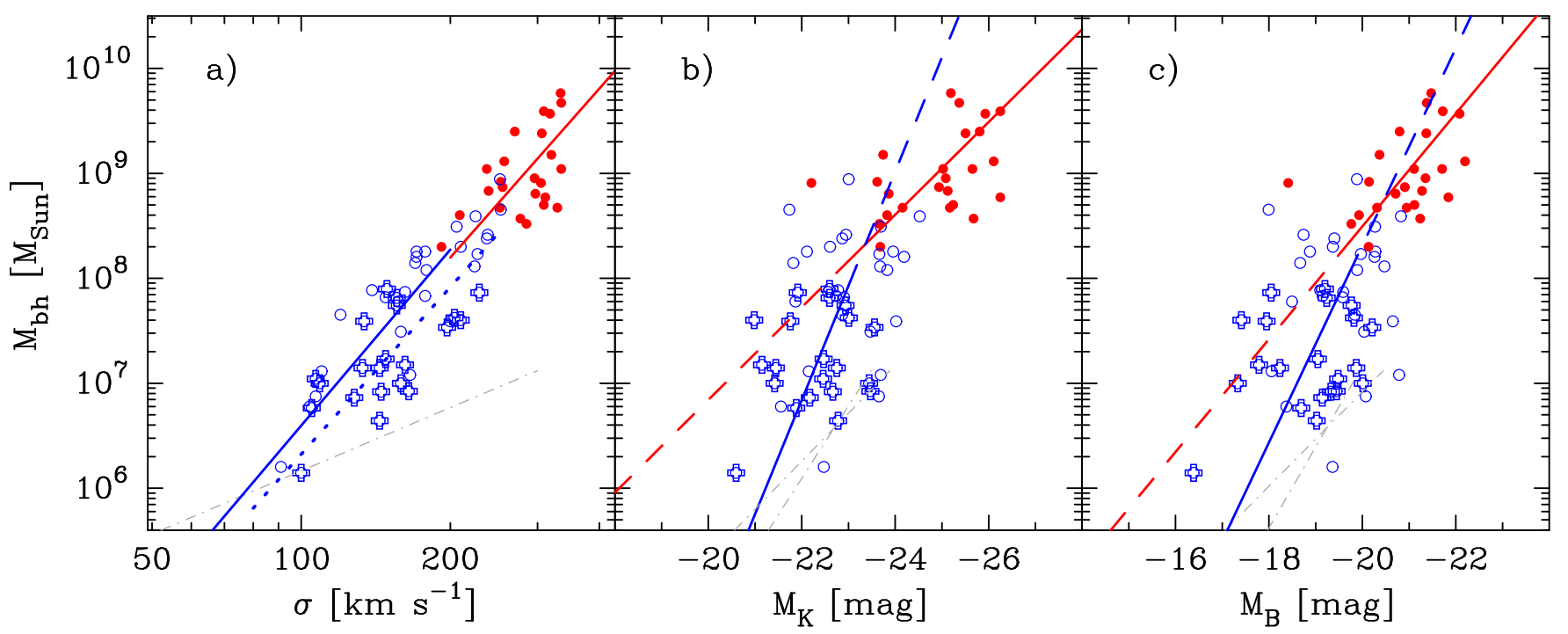

FIG. 2. - Black hole scaling relations involving a) central velocity dispersion $\sigma$, b) $K_{s}$-band host spheroid magnitude and c) $B$-band host spheroid magnitude. The red dots represent the (luminous) core-Sérsic galaxies and the blue circles represent the (intermediate-luminosity) Sérsic galaxies and bulges, while the open crosses designate those which are barred. The symmetrical bisector regressions given in Table 3 are shown here, except for panel a) which shows the $\mathrm{OLS}\left(\sigma \mid M_{\mathrm{bh}}\right)$ regression for the 21 barred (dotted blue) and 27 non-barred (solid blue) Sérsic galaxies, along with the symmetrical bisector regression for the 24 core-Sérsic galaxies. The faint dot-dashed gray line at the bottom of panel a) corresponds to a black hole's sphere-of-influence of $0^{\prime \prime} .1$ at a distance of $1.3 \mathrm{Mpc}$. With such a limiting spatial resolution of $0^{\prime \prime} .1$, black holes residing below this line cannot be reliably detected. Using the $L_{K_{s}}-\sigma$ and $L_{B}-\sigma$ relations for the Sérsic galaxies, constructed using both a bisector regression and an $\operatorname{OLS}(L \mid \sigma)$ regression, this gray line has been mapped into panels b) and c). The left-most core-Sérsic galaxy in panels b) and c) is NGC 3998.

the line which bisects the forward and inverse regression lines, having the average angle between them, is the symmetrical bisector regression line. In general, symmetrical regressions are preferred when it is not known which variable is dependent on the other, and when one wants to establish the intrinsic relation between two quantities, the so-called theorist's question (Novak et al. 2006). Biases in the data sample, due to selection boundaries, can of course bias the results from a symmetrical regression and thus create a preference for either the forward or inverse regression depending on whether the selection boundary is vertical or horizontal in one's diagram (e.g. Lynden Bell et al. 1988). Typically in the literature, the modified FITEXY routine has only been run as a 'forward' regression, minimizing the offsets in the vertical direction, where as users of the BCES code typically report the symmetrical bisector regression line. It is not appropriate to compare such results, and some confusion exists because Tremaine et al. (2002) remarked that their modified FITEXY routine treats the data symmetrically. However if this was true, if the $x$ and $y$ data was treated equally, then swapping the $x$ and $y$ data points would not alter the fit that one obtains.

Returning to our $M_{\mathrm{bh}}-L$ diagram, given the larger uncertainties on the spheroid magnitudes, compared to the uncertainties on the velocity dispersions, we have used the modified FITEXY routine to check if the BCES bisector routine has yielded a biased result. In fitting the line $y=a+b x$, Tremaine et al.'s (2002) modified version of the routine FITEXY (Press et al. 1992, their Section 15.3) minimizes the quantity

$$
\chi^{2}=\sum_{i=1}^{N} \frac{\left(y_{i}-a-b x_{i}\right)^{2}}{\delta y_{i}{ }^{2}+b^{2} \delta x_{i}{ }^{2}+\epsilon^{2}} .
$$

The intrinsic scatter (in the $y$-direction) is denoted here by the term $\epsilon$, and the measurement errors on the $N$ pairs of observables $x_{i}$ and $y_{i}$ are denoted by $\delta x_{i}$ and $\delta y_{i}$. The intrinsic scatter $\epsilon$ is solved for by repeating the fit with different values of $\epsilon$ until $\chi^{2} /(N-2)$ equals 1 . To achieve a minimization in the $x$-direction, i.e. to perform the 'inverse' regression, one can simply replace the $\epsilon^{2}$ term in the denominator with $b^{2} \epsilon^{2}$ (Novak et al. 2006; Graham 2007a; Graham \& Driver 2007a). The $M_{\mathrm{bh}}-L$ bisector line for the core-Sérsic spheroids, obtained from the bisector line of the forward and inverse modified FITEXY routine, has a slope of -0.46 and -0.56 in the $K$ - and $B$ - bands, respectively. The slope of the $M_{\mathrm{bh}}-L$ bisector line for the Sérsic spheroids, obtained from the forward and inverse modified FITEXY routine, is -0.95 and -0.88 for the $K$ and $B$-bands, respectively. These results are consistent with the values obtained from the BCES bisector routine, as reported in Table 3

\subsection{Further Pruning}

Curious readers may be wondering what happens if additional data points are pruned from the $M_{\mathrm{bh}}-L$ diagram. The 'core' galaxy with the faintest magnitude is the lenticular galaxy NGC 3998. With a velocity dispersion in excess of $300 \mathrm{~km} \mathrm{~s}^{-1}$, this galaxy is expected to be a 'core' galaxy. Figure 8 from Gonzalez Delgado et al. (2008) reveals that this galaxy hosts an AGN that swamps the central light profile and any core which may be there, hence the cautious '?' on its classification in Table 1. Re-labelling this galaxy to be a "Sersic" galaxy slightly steepens the Sersic $M_{\mathrm{bh}}-L$ relation, and hence our case for a deviation from the previous linear M-L relation, but not significantly. To the immediate lower left of this galaxy in the $M_{\mathrm{bh}}-L$ diagram is NGC 4342. This Sérsic galaxy was labelled an outlier by Laor (2001) and a potential outlier by Häring \& Rix (2004), both of whom excluded it from their analysis. Bogdán et al. (2012a) have also identified it as unusual, and it may be a heavily stripped galaxy (Blom et al. 2012), causing it to deviate 


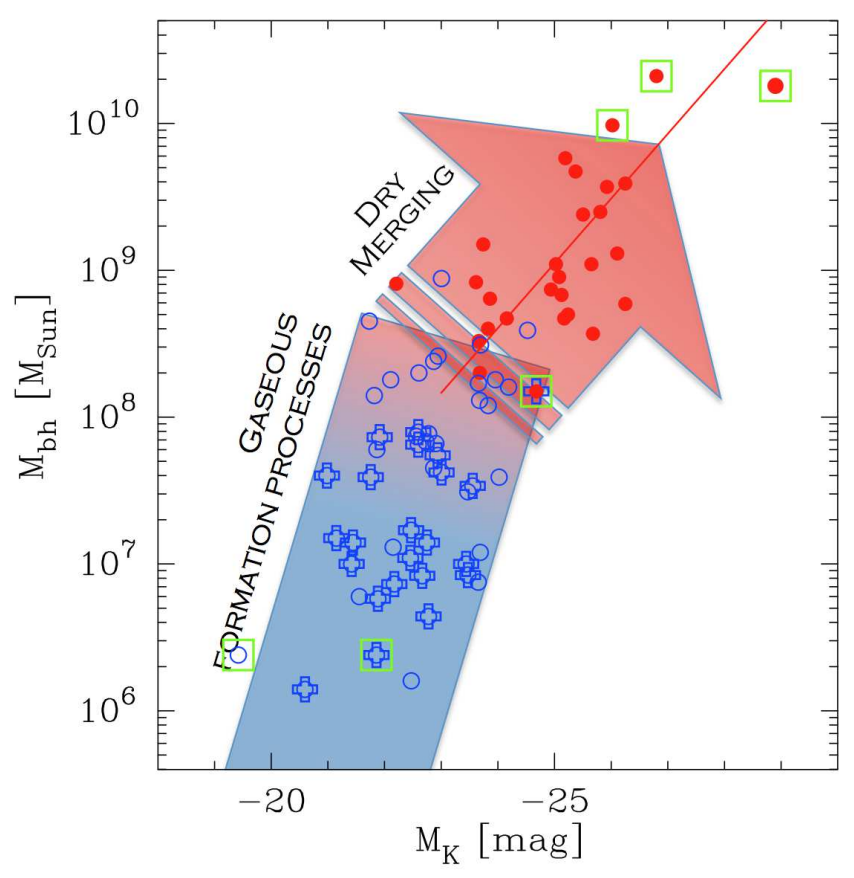

FIG. 3.- Schematic view of Figure 2 2 showing the evolutionary path of 'dry' galaxy mergers as they branch off from the steeper, near-quadratic, $M_{\mathrm{bh}}-L$ relation for Sérsic galaxies built from 'wet' mergers and/or gas-rich processes. The upper red arrow roughly shows the direction of growth if $M_{\mathrm{bh}} \propto L^{1.0}$, while the slope of the blue shaded area roughly follows $M_{\mathrm{bh}} \propto L^{2.5}$. An additional five galaxies (NGC 3842 and NGC 4889 from McConnell et al. 2011a, NGC 1316, NGC 3079 and M32), which were excluded from the regression analysis in Figure 2] are shown here, along with OJ278 from Valtonen et al. (2012), and marked with a green square.

from the $M_{\mathrm{bh}}-L$ relation while still following the $M_{\mathrm{bh}}-$ $\sigma$ relation. M32 may be another such stripped galaxy (Bekki 2001; Graham 2002). Excluding NGC 3998 and NGC 4342 results in an $M_{\mathrm{bh}}-L_{K_{s}}$ relation with slopes of $-0.50 \pm 0.08$ and $-1.07 \pm 0.24$ for the Sérsic and coreSérsic galaxies, respectively.

\section{DISCUSSION AND IMPLICATIONS}

The coevolution of black holes and their host spheroids has resulted in a bent, i.e. a non-(log-linear), $M_{\mathrm{bh}}-L$ relation which can be approximated by a broken power-law having an exponent of $\sim 1$ for spheroids with core-Sérsic profiles and $\sim 2.5$ for spheroids with Sérsic profiles. The major, i.e. near equal mass, merger of gas-free galaxies near the bright-end of the Sérsic sequence will preserve the $M_{\mathrm{bh}} / L$ ratio and create the core-Sérsic galaxies, which are observed to follow a near linear $M_{\mathrm{bh}}-L$ relation. That is, such dry mergers do not move galaxies along the steep $M_{\mathrm{bh}}-L$ relation defined by Sérsic galaxies, but drive them off it to form a distinctly different sequence (Figure 31 ${ }^{12}$. The above picture (and Figure 3) suggests that the bulk of AGN emission, i.e. black hole growth through gas-rich processes, should be associated with black holes having masses less than a few times $10^{8} M_{\odot}$. The peak of the Eddington ratio at $M_{\mathrm{bh}}=(4-$ $8) \times 10^{7} M_{\odot}$ is not inconsistent with this (DeGraf et al. 2012). Magnitude-limited high-redshift quasar surveys may thus only be probing the tip of the proverbial ice-

\footnotetext{
12 A substantial gas-rich merger with, or gas inflow onto, a coreSérsic galaxy may drive it above the one-to-one relation seen in the $M_{\mathrm{bh}}-L$ diagram.
}

berg.

An exponent of 1 for the core-Sérsic galaxies in the $M_{\mathrm{bh}}-L$ diagram would mean that there is a constant $M_{\mathrm{bh}} / M_{\text {spheroid }}$ mass ratio for these galaxies. For a coreSérsic spheroid with a (dust-free) absolute magnitude $M_{K_{s}}=-25.33 \mathrm{mag}$, and assuming an $M / L_{K}$ ratio of 0.8 (Bell \& de Jong 2001) and a solar $K$-band absolute magnitude of $3.27 \mathrm{mag}$ (Cox 2000), we find a median mass ratio $M_{\mathrm{bh}} / M_{\mathrm{Sph}, *}=0.49 \%$. For a core-Sérsic spheroid with a (dust-free) absolute magnitude $M_{B}=-21 \mathrm{mag}$, and assuming an $M / L_{B}$ ratio of 5 and a solar $B$-band absolute magnitude of $5.47 \mathrm{mag}$ (Cox 2000), we find a similar (black hole)-to-(stellar) mass ratio of $0.55 \%$. These ratios are larger than the old median value of 0.14 $0.2 \%$ (e.g. Ho 1999; Marconi \& Hunt 2003; Häring \& Rix 2004) for the (black hole)-to-(spheroid dynamical) mass ratio. Even if we assumed $M / L_{B}=8$, we find $M_{\mathrm{bh}} / M_{\mathrm{Sph}, *}=0.35 \%$.

The steep exponent of $\sim 2.5$ for the Sérsic galaxies in the $M_{\mathrm{bh}}-L$ diagram means that the pre-existing single log-linear $M_{\mathrm{bh}}-L$ relation will overestimate the black hole masses in galaxies whose black hole mass is less than $\sim 10^{8} M_{\odot}$. This is in fact already evident in Khorunzhev et al. (2012) who found that $M_{\mathrm{bh}}$ was overestimated using the old $M_{\mathrm{bh}}-L$ relations for $M_{\mathrm{bh}}<10^{8} M_{\odot}$, just where the bend in the $M_{\mathrm{bh}}-L$ relation sets in. Similarly, Mathur et al. (2012, their figure 3) show ten galaxies below the old $M_{\mathrm{bh}}-L$ relation at $\log \left(M_{\mathrm{bh}}\right)=7 \pm 0.4$. However their interpretation that these galaxies host pseudobulges, based on their location in the $M_{\mathrm{bh}}-L$ diagram, is premature because these galaxies are found where the classical bulges and elliptical galaxies reside.

Having the correct black hole scaling relations is, obviously, important when trying to predict a galaxy's black hole mass. Equally important is having the correct velocity dispersion or bulge magnitude. In regard to the core-Sérsic galaxy NGC 4382 (Dullo \& Graham 2012), Gültekin et al. (2011) predicted a black hole mass that differed by an order of magnitude when one used the $M_{\mathrm{bh}}-L$ and $M_{\mathrm{bh}}-\sigma$ relations (Gültekin et al. 2009b). Here we predict $\log \left(M_{\mathrm{bh}} / M_{\odot}\right)=8.0 \pm 0.3$ when using $\sigma=182 \pm 5 \mathrm{~km} \mathrm{~s}^{-1}$ (Gültekin et al. 2011), in agreement with Gültekin et al. (2011). However this galaxy appears to be a disturbed, face-on, non-barred, lenticular galaxy (Laurikainen et al. 2011) and we therefore do not use the total galaxy light to estimate its central black hole mass. The total $2 \mathrm{MASS} K_{s}$-band magnitude of $6.145 \mathrm{mag}$ corresponds to an absolute magnitude of roughly $-25.1 \mathrm{mag}$ given a distance modulus of 31.27 (Tonry et al. 2001; Blakeslee et al. 2002). Galactic extinction and redshift dimming are negligible. Using an average, dust-corrected, S0 galaxy bulge-to-disk flux ratio of one third, we thus have a $K_{s}$-band bulge magnitude of -23.9 mag. Using the $M_{\mathrm{bh}}-L_{K_{s}}$ relation in Table 3 , we find $\log \left(M_{\mathrm{bh}} / M_{\odot}\right)=8.6 \pm 0.5$ when using a factor of 2 uncertainty on this bulge magnitude. This estimated black hole mass has over-lapping error bars with the first estimate. We would however predict a black hole mass of $\sim 10^{9} M_{\odot}$, in agreement with Gültekin et al. (2011), if all of the light in this disturbed galaxy was assigned to a single spheroid component.

Having the right $M_{\mathrm{bh}}-L$ relation is of course also important when using the galaxy/spheroid luminosity function to predict the black hole mass function, and sub- 
sequently the black hole mass density (e.g. Graham \& Driver 2007b, and references therein). Knowledge of the correct $M_{\mathrm{bh}}-L$ relation will additionally be useful for cosmological / evolutionary studies of the nucleus-to(host spheroid) mass ratio (e.g. Kisaka \& Kojima 2010; Schulze \& Wisotzki 2011; Portinari et al. 2012; Sesana 2012), and for many other studies. The consequences of the new bent $M_{\mathrm{bh}}-L$ and bent $M_{\mathrm{bh}}-M_{\text {spheroid }}$ relations are widespread and dramatic. Below we discuss some of these in more detail.

\subsection{Intermediate mass black holes}

Classical elliptical galaxies with $M_{B} \approx-18$ mag will now have $\left(M_{\mathrm{bh}}-L\right)$-derived black hole masses which are ten times lower than previously predicted. Similarly, we also now have revised predictions for the location of $10^{6} M_{\odot}$ black holes within the $M_{\mathrm{bh}}-L$ diagram. Based on their host spheroid luminosity, they will have black hole masses which are roughly 10 times lower than predicted from the extrapolation of the $M_{\mathrm{bh}}-L$ relation defined by luminous core-Sérsic galaxies, placing them near the current detection limit shown in Figure 2, Black holes with masses of $10^{5}-10^{6} M_{\odot}$ will be even further from the extrapolated core-Sérsic $M_{\mathrm{bh}}-L$ relation. A number of AGN residing in elliptical galaxies, not to be confused with the bulges of disk galaxies, have been observed below this relation. For example, Greene et al. (2008) showed some AGN which had black hole masses that are an order of magnitude below both the old $M_{\mathrm{bh}}-L$ relation and the $M_{\mathrm{bh}}-M_{\text {spheroid }}$ relation from Häring \& Rix (2004). Our interpretation of this offset differs from Greene et al. (2008), in that we consider their sample of non-disk galaxies to be the extension of the Sérsic sequence, rather than a separate galaxy population which Greene et al. (2008) refer to as "blobs".

\subsubsection{Individual galaxies}

Interestingly, the $\left(M_{\mathrm{bh}}-M_{\text {spheroid }}\right)$-derived mass determination (using the expression from Häring \& Rix 2004) for the black hole associated with the recent ultravioletoptical flare from the tidal disruption of a helium-rich stellar core in a galaxy at a redshift of 0.17 (Gezari et al. 2012) can now be revised. Using the updated $M_{\mathrm{bh}}-M_{\text {spheroid }}$ relation from Graham (2012a), its black hole mass is $\sim 5$ times lower than the reported value of $4 \times 10^{6} M_{\odot}$, making it slightly less than one million solar masses.

Our revised black hole scaling relations suggest that the high-energy transient Sw 1644+57 (Levan et al. 2011; Bloom et al. 2011) may have been associated with a stellar capture event by a black hole having a mass of $0.6 \times 10^{6} M_{\odot}$ (and even less if the host galaxy is a disk galaxy). Not only does the host galaxy's absolute magnitude $\left(M_{V}=-18.19 \mathrm{mag}\right)$ suggest this low black hole mass (for $B-V=0.9$ ), but so does the reported host galaxy mass of a few billion solar masses (Bloom et al. 2011) when using the $M_{\mathrm{bh}}-M_{\text {spheroid }}$ relation from Graham (2012a). We therefore tentatively claim the presence of an intermediate mass black hole in this galaxy, adding to the detection of other intermediate mass black hole candidates (e.g., Farrell et al. 2009; Soria et al. 2010; Sutton et al. 2012).

The revised $M_{\mathrm{bh}}-L$ scaling relations presented here give rise to the need to re-derive many past estimates of black hole masses, in particular those associated with active galactic nuclei in low luminosity bulges. Whether or not these objects can provide unique observational constraints on the progenitors of supermassive black holes is unclear, but they do narrow the divide between today's supermassive black holes and their 'seeds'.

For the Seyfert Sc galaxy NGC 3367 (McAlpine et al. 2011), Dong \& De Robertis (2006) report a $K$-band bulge magnitude of $-21.6 \mathrm{mag}$, corresponding to a bulge-tototal flux ratio of 0.08. Hernández-Toledo et al. (2011) find that the bulge has a Sérsic index between 1 and 1.8 , and after including a bar in their image analysis they report a bulge-to-disc ratio of 0.07 to 0.11 . The bulge magnitude from Dong \& De Robertis (2006), after brightening it by $0.11 \mathrm{mag}$ for dust (Driver et al. 2008), yields an expected black hole mass of $\log M_{\mathrm{bh}} / M_{\odot}=$ $6.2 \pm 0.9$. McAlpine et al. (2011) also studied the Sbc galaxy NGC 4536, reported to have a bulge Sérsic index of $1.88 \pm 0.35$ by Fisher \& Drory (2008), and an absolute $V$-band magnitude of $-18.4 \mathrm{mag}$ assuming a distance of $25.3 \mathrm{Mpc}$. With a disk inclination of 62 degrees (Fisher \& Drory 2008) we apply a dust correction of $1.3 \mathrm{mag}$ (Driver et al. 2008), and using an assumed $B-V$ bulge color of 0.8 we have that $M_{B}=-18.9 \mathrm{mag}$. The expected black hole mass is thus $\log M_{\mathrm{bh}} / M_{\odot}=7.3 \pm 0.8$, assuming a 0.3 mag uncertainty on the bulge magnitude. These two galaxies are not, therefore, expected to house low intermediate mass black holes.

If the dwarf galaxy Pox 52 - long thought to house an AGN (Kunth, Sargent \& Bothun 1987) — is an elliptical galaxy, then its $B$-band magnitude of $-16.8 \mathrm{mag}$ (Barth et al. 2004) translates into an expected black hole mass of $\log M_{\mathrm{bh}} / M_{\odot}=5.3 \pm 0.9$, with the uncertainty of 0.5 dex derived assuming an uncertainty of $0.3 \mathrm{mag}$ on the galaxy magnitude.

For the nearby dwarf lenticular galaxy NGC 404, the Galactic extinction corrected $H$-band magnitude of $-19.65 \mathrm{mag}$ from Seth et al. (2010) becomes $M_{K_{s}}=$ $-19.85 \mathrm{mag}$ when using an $H-K_{s}$ color of $0.2 \mathrm{mag}$ (e.g. Balcells et al. 2007). Thought to house an AGN (e.g. Nyland et al. 2012, and references therein), the expected black hole mass in NGC 404 is $\log M_{\mathrm{bh}} / M_{\odot}=4.1 \pm 1.1$, using a magnitude error of 0.3 mag. For comparison, although restricted by assumptions about the inner stellar mass-to-light ratio, the stellar dynamical modeling from Seth et al. (2010) suggests that NGC 404 has a black hole mass less than $10^{5} M_{\odot}$.

The bulge of the Scd galaxy NGC 1042 has a dust corrected magnitude of $-19.13 \pm 0.75 \mathrm{~K}$-mag (Knapen et al. 2003; Graham \& Worley 2008) and houses an active black hole (Shields et al. 2008; Seth et al. 2008). From the new $K$-band $M_{\mathrm{BH}}-L_{\text {bulge }}$ relation, the expected black hole mass is $\log M_{\mathrm{bh}} / M_{\odot}=3.3 \pm 1.4$.

Given a $B$-band bulge magnitude of -13.93 mag (Graham \& Spitler 2009) for the AGN-hosting galaxy NGC 4395 (Filippenko \& Ho 2003), the expected black hole mass in this late-type galaxy's bulge is equally small. Assuming a $0.75 \mathrm{mag}$ uncertainty on the bulge magnitude, the black hole mass is $\log M_{\mathrm{bh}} / M_{\odot}=2.6 \pm 1.3$. Allowing for the 1-sigma uncertainty given here, this mass resides just below the $10^{4}-10^{5} M_{\odot}$ mass range estimated by Filippenko \& Ho (2003) and is also below the (reverberation mapping)-derived estimate of $3.6 \pm 2.2 \times 10^{5} M_{\odot}$ (Peterson et al. 2005), even after it has been halved to 
TABLE 5

SUPERMASSIVE AND INTERMEDIATE MASS BLACK HOLES

\begin{tabular}{lll}
\hline Ident. & magnitude & $\log M_{\mathrm{bh}}$ \\
\hline NGC 4536 & $-18.9 B$-mag & $7.3 \pm 0.8$ \\
NGC 3367 & $-21.71 K$-mag & $6.2 \pm 0.9$ \\
Gezari et al. & $\ldots$ & $5.9 \pm 0.9$ \\
Sw 1644+57* & $-18.19 \mathrm{~V}$-mag & $5.8 \pm 0.9$ \\
Pox 52 & $-16.8 B$-mag & $5.3 \pm 0.9$ \\
NGC 404 & $-19.85 K$-mag & $4.1 \pm 1.1$ \\
NGC 1042 & $-19.13 K$-mag & $3.3 \pm 1.4$ \\
NGC 4395 & $-13.93 B$-mag & $2.6 \pm 1.3$ \\
\hline
\end{tabular}

* This transient's designation is used to identify the host galaxy.

$1.8 \pm 0.6 \times 10^{5} M_{\odot}$ due to the updated virial $f$-factor in Graham et al. (2011). A lower black hole mass is however expected from the short time-scale variations of the X-ray flux which can vary by an order of magnitude (Vaughan et al. 2005), and would of course necessitate a higher Eddington accretion rate than the previously assumed maximum of just a couple of percent.

All of our black hole mass estimates above have been collated in Table 5 for ease of reference and overview.

Typically, when X-ray flux is detected in galaxies having a candidate intermediate mass black hole, the level of emission is so low that skeptics point to possible stellar origins for its production. That is, in general, there is not the danger that such galaxies might have too much X-ray luminosity supportive of a supermassive black hole rather than an intermediate mass black hole.

\subsubsection{A catalog of galaxies: Dong $\mathscr{G}$ De Robertis}

In addition to the above mentioned galaxies which have had individual papers dedicated to them, we are able to identify many other intermediate mass black hole candidates from a catalog of low-luminosity AGN. The loglinear $K_{s}$-band $M_{\mathrm{bh}}-L$ relation of Dong \& De Robertis (2006), which was consistent with that from Marconi \& Hunt (2003), was used by Dong \& De Robertis to predict the masses of black holes in 117 disc galaxies hosting lowluminosity AGN. From their sample of bulge luminosities, they reported only 4 galaxies with estimated black hole masses less than $10^{6} M_{\odot}$ and such that $\log M_{\mathrm{bh}} / M_{\odot}$ $=5.6$ to 5.8. Given this, coupled with the uncertainty in the $M_{\mathrm{bh}}-L$ relation, they understandably paid no attention to the possible detection of intermediate mass black holes in their sample. However given the dramatically steeper $M_{\mathrm{bh}}-L$ relation reported in this paper for intermediate- and low-luminosity spheroids, we expect that many of the black hole mass estimates in Dong \& De Robertis will have been overestimated. As a consequence, we note in passing that the associated scaling relations reported there between black hole mass and line width (FWHM [N II]), as well as emission-line ratios (e.g., $[\mathrm{O} \mathrm{III}] / \mathrm{H} \beta,[\mathrm{O} \mathrm{I}] / \mathrm{H} \alpha,[\mathrm{N} \mathrm{II}] / \mathrm{H} \alpha$, and $[\mathrm{S} \mathrm{II}] / \mathrm{H} \alpha$ ), may need to be re-derived.

Here we predict new black hole masses for the 41 galaxies reported by Dong \& De Robertis (2006, their Table 4 ) to have absolute $K_{s}$-band bulge magnitudes fainter than -21.0 mag. Before applying our Sérsic $M_{\mathrm{bh}}-L$ relation, we correct (brighten) these bulge magnitudes for internal dust extinction. This has been done using the inclination-dependent, dust correction from Driver et al. (2008), and is shown in Table 6. The predicted black hole masses shown there assumes that the $M_{\mathrm{bh}}-L$ rela- tion can be extrapolated to lower black hole masses, and the quoted error was derived assuming an uncertainty on the magnitudes of $0.3 \mathrm{mag}$.

The two lowest mass, intermediate mass black hole candidates in Table 6 are the barred galaxies NGC $4136\left(\log M_{\mathrm{bh}} / M_{\odot}=2.2 \pm 1.3\right)$ and NGC 3756 $\left(\log M_{\mathrm{bh}} / M_{\odot}=2.6 \pm 1.3\right)$. We can apply our updated $M_{\mathrm{bh}}-\sigma$ relation for barred galaxies, shown in bold in Table 3. although a serious concern is that the available velocity dispersions of $38.4 \pm 8.7$ and $47.6 \pm 8.5$ (Ho et al. 2009) may be elevated by the velocity dispersion of the disk given the low bulge luminosities. The respective $\left(M_{\mathrm{bh}}-\sigma\right)$-derived masses $\log M_{\mathrm{bh}} / M_{\odot}=4.13 \pm 1.26$ and $4.62 \pm 1.11$ do however still have over-lapping error bars with the $\left(M_{\mathrm{bh}}-L\right)$-derived masses.

Finally, we note that moving down the spheroid luminosity function, black holes appear to give way to, or at least co-exist with, dense nuclear star clusters (e.g. Graham \& Spitler 2008; Graham 2012b; Neumayer \& Walcher 2012; Leigh, Böker \& Knigge 2012; Scott \& Graham 2012). It is not clear to what lower masses our Sérsic $M_{\mathrm{bh}}-L$ relation may hold. We do however note that many new intermediate mass black hole candidates, with $M_{\mathrm{bh}}=0.8 \times 10^{5}-10^{6} M_{\odot}$, measured from virial mass estimator based on the broad $\mathrm{H} \alpha$ line, have recently been identified (Dong et al. 2012).

\subsection{The $M_{\mathrm{bh}}-M_{\text {host-galaxy }}$ connection}

For early-type galaxies, the dynamical mass-to-light ratio is thought to roughly change with the optical spheroid luminosity as $(M / L)_{\text {dynamical }} \propto L^{1 / 4}$ (e.g. Faber et al. 1987; Cappellari et al. 2006, their eq. 17) or rather $\propto L^{1 / 3}$ (Cappellari et al. 2006, their eq.9 and 11), while $M / L$ should obviously be constant for the core-Sérsic galaxies if built via dry (additive) mergers. That is, the core-Sérsic $M_{\mathrm{bh}}-M_{\mathrm{Sph} \text {,dyn }}$ relation should have the same (close to unity) slope as the core-Sérsic $M_{\mathrm{bh}}-L_{\text {spheroid }}$ relations $M_{\mathrm{bh}} \propto L_{B}^{1.35 \pm 0.30}$ and $M_{\mathrm{bh}} \propto L_{K_{s}}^{1.10 \pm 0.20}$. However we need to caution that galaxy samples with a greater fraction of core-Sérsic galaxies relative to Sérsic galaxies may have in the past resulted in a shallower average $(M / L)_{\text {dynamical }} \propto L$ relation than is actually applicable to the Sérsic galaxies on their own. Studies of the Fundamental Plane (Djorgovski \& Davis 1987) in the nearinfrared $J$ - and $K$-bands, which are less affected by stellar population differences across the galaxy luminosity range, have tended to yield $(M / L)_{\text {dynamical }} \propto L_{K}^{1 / 6}$ (e.g. Magoulas et al. 2012; La Barbera et al. 2010), although the expanded SAURON survey with $72 \mathrm{E} / \mathrm{S} 0 / \mathrm{Sa}$ galaxies reported $(M / L)_{\text {dynamical }} \propto L_{V}^{1 / 3}$ and $\propto L_{3.6 \mu}^{1 / 4}$ (FalcónBarroso et al. 2011). The ATLAS ${ }^{3 \mathrm{D}}$ survey (Cappellari et al. 2011) is comprised of just a few percent of core-Sérsic galaxies supported by random motions rather than rotational velocity (Emsellem et al. 2011) and it will therefore be interesting to see if they also find these steeper $M / L-L$ relations.

For now, using the shallower $M / L$ relations above, which admittedly may underestimate the slope of the Sérsic galaxy $(M / L)_{\text {dynamical }} \propto L$ relation, our Sérsic $M_{\mathrm{bh}}-L_{\text {spheroid }}$ expressions can be roughly transposed into (black hole)-(host spheroid) mass relations. One has that $M_{\mathrm{bh}} \propto L_{B}^{2.35 \pm 0.40}$ maps into $M_{\mathrm{bh}} \propto M_{\mathrm{Sph}, \mathrm{dyn}}^{1.88 \pm 0.32}$ 
The $M_{\mathrm{bh}}-L_{\text {spheroid }}$ relation

TABLE 6

Predicted InTERMEdiate Mass BLACK HOLES

\begin{tabular}{lccl}
\hline Galaxy & $M_{K_{s}, \text { orig }}$ & $M_{K_{s}, \text { corr }} \log M_{\mathrm{bh}} / M_{\odot}$ \\
\hline NGC 3185 & -20.80 & -20.94 & $5.3 \pm 0.9$ \\
NGC 3593 & -20.70 & -21.03 & $5.4 \pm 0.9$ \\
NGC 3600 & -19.20 & -19.72 & $4.0 \pm 1.1$ \\
NGC 3729 & -20.10 & -20.24 & $4.6 \pm 1.0$ \\
NGC 4245 & -20.80 & -20.93 & $5.3 \pm 0.9$ \\
NGC 4314 & -21.00 & -21.11 & $5.5 \pm 0.9$ \\
NGC 4369 & -20.90 & -21.01 & $5.4 \pm 0.9$ \\
NGC 4470 & -20.40 & -20.53 & $4.9 \pm 1.0$ \\
NGC 3003 & -19.60 & -20.09 & $4.4 \pm 1.0$ \\
NGC 3043 & -20.80 & -21.17 & $5.6 \pm 0.9$ \\
NGC 3162 & -20.00 & -20.12 & $4.4 \pm 1.0$ \\
NGC 3344 & -19.30 & -19.41 & $3.7 \pm 1.1$ \\
NGC 3507 & -20.90 & -21.01 & $5.4 \pm 0.9$ \\
NGC 3684 & -20.60 & -20.74 & $5.1 \pm 1.0$ \\
NGC 3686 & -20.60 & -20.72 & $5.1 \pm 1.0$ \\
NGC 3756 & -18.20 & -18.43 & $2.6 \pm 1.3$ \\
IC 467 & -19.20 & -19.50 & $3.8 \pm 1.1$ \\
NGC 514 & -19.90 & -20.02 & $4.3 \pm 1.0$ \\
NGC 628 & -20.40 & -20.51 & $4.9 \pm 1.0$ \\
NGC 864 & -19.90 & -20.03 & $4.3 \pm 1.0$ \\
NGC 2276 & -20.90 & -21.01 & $5.4 \pm 0.9$ \\
NGC 2715 & -19.20 & -19.56 & $3.8 \pm 1.1$ \\
NGC 2770 & -19.10 & -19.50 & $3.8 \pm 1.1$ \\
NGC 2776 & -20.90 & -21.01 & $5.4 \pm 0.9$ \\
NGC 2967 & -20.30 & -20.41 & $4.8 \pm 1.0$ \\
NGC 3041 & -19.90 & -20.05 & $4.4 \pm 1.0$ \\
NGC 3198 & -19.80 & -20.11 & $4.4 \pm 1.0$ \\
NGC 3359 & -20.80 & -20.97 & $5.4 \pm 0.9$ \\
NGC 3430 & -20.10 & -20.29 & $4.6 \pm 1.0$ \\
NGC 3433 & -20.40 & -20.51 & $4.9 \pm 1.0$ \\
NGC 3486 & -19.90 & -20.03 & $4.3 \pm 1.0$ \\
NGC 3596 & -21.00 & -21.11 & $5.5 \pm 0.9$ \\
NGC 3666 & -18.20 & -18.63 & $2.8 \pm 1.2$ \\
NGC 3726 & -20.10 & -20.24 & $4.6 \pm 1.0$ \\
NGC 3780 & -20.20 & -20.32 & $4.7 \pm 1.0$ \\
NGC 3938 & -20.50 & -20.61 & $5.0 \pm 1.0$ \\
NGC 4062 & -18.50 & -18.78 & $3.0 \pm 1.2$ \\
NGC 4096 & -19.40 & -19.84 & $4.1 \pm 1.1$ \\
NGC 4136 & -18.00 & -18.11 & $2.2 \pm 1.3$ \\
NGC 4152 & -20.80 & -20.92 & $5.3 \pm 0.9$ \\
NGC 4212 & -20.10 & -20.27 & $4.6 \pm 1.0$ \\
\hline Is $99795,-b a n$ \\
NGp
\end{tabular}

$M_{K_{s}, \text { orig }}$ is each galaxy's $K_{s}$-band, spheroid magnitude reported by Dong \& De Robertis (2006). $M_{K_{s}, \text { corr }}$ is the dust-corrected magnitude following Driver et al. (2008). $M_{\mathrm{bh}}$ is our predicted black hole mass using the Sérsic $M_{\mathrm{bh}}-L_{K_{s}}$ relation from Table 4 (For reference, Dong \& De Robertis (2006) predicted only 4 galaxies to have black hole masses less than $10^{6} M_{\odot}$, which were in the range $\log M_{\mathrm{bh}} / M_{\odot}=5.6$ to 5.8.)

(when using $M / L_{B} \propto L_{B}^{1 / 4}$ ) and $M_{\mathrm{bh}} \propto L_{K_{s}}^{2.73 \pm 0.55}$ maps into $M_{\mathrm{bh}} \propto M_{\mathrm{Sph}, \mathrm{dyn}}^{2.34 \pm 0.47}$ (when using $M / L_{K_{s}} \propto$ $\left.L_{K_{s}}^{1 / 6}\right)$. This can be directly compared with the slopes of $1.01 \pm 0.52$ for the core-Sérsic galaxies in Graham (2012a), and $2.30 \pm 0.47$ and $1.92 \pm 0.38$ for the Sérsic and nonbarred Sérsic galaxies in that study.

Rather than described by a single power-law (e.g. Magorrian et al. 1998), the full $M_{\mathrm{bh}}-M_{\mathrm{Sph}, \mathrm{dyn}}$ relation appears to be better described by a broken power-law having exponents of $\sim 1$ for the core-Sérsic spheroids and $\sim 2$ for the Sérsic spheroids, in agreement with the previous announcement by Graham (2012a) based on a reanalysis of the Häring \& Rix (2004) data (see also Balcells et al. 2007, their equation 13). The non-linear, albeit still a single log-linear, (black hole)-(host stellar mass) relation from Laor (2001) was given as $M_{\mathrm{bh}} \propto$ $M_{\mathrm{Sph}, *}^{1.53 \pm 0.14}$ and was not a mistake but rather arose from fitting a sample of what are undoubtedly Sérsic and coreSérsic spheroids.

If, as commonly believed, the core-Sérsic galaxies are built from the simple addition of high-mass gas-free Sérsic galaxies and/or other core-Sérsic galaxies, then one may similarly expect $M_{\mathrm{bh}}$ to be linearly correlated with the dark halo mass in core-Sérsic galaxies (Ferrarese 2002; Bogdán et al. 2012a). Of course this (noncausal) relation will not hold across the full mass range of Sérsic spheroids (Ho 2007). The single power-law relation involving the mass of the central black hole and the surrounding dark matter halo which was derived for a sample of both Sérsic and core-Sérsic galaxy types, $M_{\mathrm{bh}} \propto M_{\mathrm{DM}}^{1.65-1.82}$ (Ferrarese 2002), may be better described by a broken power-law - at least over the highmass range where a correlation exists. Looking at Figure 5 from Ferrarese (2002), galaxies with $M_{\mathrm{bh}} \gtrsim 10^{8} M_{\odot}$ appear to be better described by a relation with an exponent closer to 1 than 1.65-1.82, with a quick analysis of their Figure revealing that an exponent of $\approx 1.2$ provides a fair fit. This is close to the values of 1.10 and 1.35 from our relations $M_{\mathrm{bh}} \propto L_{K_{s}}^{1.10 \pm 0.20}$ and $M_{\mathrm{bh}} \propto L_{B}^{1.35 \pm 0.30}$. Thankfully complex models involving the growth of seed black holes in high-redshift dark matter halos are not required to explain this near-linear relation if it is created by the simple addition of similar galaxies.

\subsection{Black hole growth in semi-analytical models}

Given the log-linear relation between Sérsic index $n$ and spheroid luminosity L (e.g. Graham \& Guzmán 2003, and references therein), the bent $M_{\mathrm{bh}}-n$ relation (Graham \& Driver 2007a) necessitates that the $M_{\mathrm{bh}}-L$ relation must, as observed here, also be bent. It would appear that black holes within spheroids formed from wet-mergers and merger triggered accretion episodes (Sanders \& Mirabel 1996; Villar-Martín et al. 2012), and/or monolithic-like collapse - especially for isolated systems - grew via quadratic black hole growth such that the $M_{\mathrm{bh}}-M_{\text {spheroid }}$ relation has a power-law slope of 2 for the Sérsic spheroids. That is, the black hole should grow more quickly than the population of stars in the host spheroid, and this has in fact recently been observed (Seymour et al. 2012; see also Greene et al. 2009).

Following Kauffmann \& Haehnelt (2000), numerous theories and models (e.g. Hopkins et al. 2006; Johansson, Naab \& Burkert 2009; Cen 2012; Hirschmann et al. 2012) have been developed to create or match a near-linear $M_{\mathrm{bh}}-L$ and $M_{\mathrm{bh}}-M_{\text {spheroid }}$ relation. Unfortunately because of this, some of their claimed 'success' implies that they are actually in need of refinement. However some models may contain important elements or clues that have been somewhat over-looked by the community. For example, the models of Dubois et al. (2012) reveal that while the $M_{\mathrm{bh}}-\sigma$ relation is linear and has a steeper slope than 4 , their simulated $M_{\mathrm{bh}}-L$ relation is bent at around $M_{\mathrm{bh}}=10^{8} M_{\odot}$, as is the simulated $M_{\mathrm{bh}}-M_{\text {Spheroid }}$ relation of Cirasuolo et al. $(2005)^{13}$. The hydrodynamical simulation models of Khandai et al. (2012, see their figure 7 ) clearly reveal a steep $M_{\mathrm{bh}}-M_{\text {spheroid }}$ relation such

13 Cirasuolo et al. (2005) additionally predict a steepening of the $M_{\mathrm{bh}}-\sigma$ relation at lower black hole masses. 
that black hole growth outpaces that of the host spheroid, in agreement with Graham (2012a) and this study.

The primary, so-called 'quasar' or 'cold', mode of black hole growth during gas-rich mergers / processes currently assumes, in semi-analytical models (Kauffmann \& Haehnelt 2000, their eq 2; Croton et al. 2006, their eq. 8; Guo et al. 2011, their eq. 36) ${ }^{14}$, that black hole growth occurs via accretion which is linearly proportional to the inflowing mass of cold gas (which also produces the host spheroid), modulated by an efficiency which is lower for both unequal mass mergers (Croton et al. 2006) and less massive (more gas-rich) systems with lower virial velocities. We therefore offer the following new prescription for the increase in black hole mass, $\delta M_{\mathrm{bh}}$, due to gas accretion during wet mergers:

$$
\delta M_{\mathrm{bh}} \propto\left(\frac{M_{\mathrm{min}}}{M_{\mathrm{maj}}}\right)\left[\frac{M_{\mathrm{cold}}^{2}}{1+\left(280 \mathrm{~km} \mathrm{~s}^{-1}\right) / V_{\text {virial }}}\right] .
$$

Here, $M_{\text {min }}$ and $M_{\text {maj }}$ are the total baryonic masses from the minor and major galaxies involved, and $M_{\text {cold }}$ is their combined cold gas mass. The term $V_{\text {virial }}$ is the circular or 'virial' velocity of the newly wed galaxy's halo, normalized at $280 \mathrm{~km} \mathrm{~s}^{-1}$ following Kauffmann \& Haehnelt (2000). This expression, now with an exponent of 2 on the cold gas mass, assumes quadratic growth for the black hole relative to the stellar mass of the host spheroid.

Another consequence of this order-of-magnitude lower $M_{\mathrm{bh}} / M_{\text {spheroid }}$ mass ratio in faint spheroids is that AGN feedback, and in particular the ratio of this feedback energy $\left(\propto M_{\mathrm{bh}}\right)$ to the binding energy of the spheroid $\left(\propto M_{\text {spheroid }} \sigma^{2}\right)$, will be an order of magnitude less than previously assumed by studies which used the old constant value of $M_{\mathrm{bh}} / M_{\text {spheroid }} \approx 0.002$ (Marconi \& Hunt 2003; Häring \& Rix 2004).

\subsection{Barred galaxies}

As noted earlier, barred galaxies can reside up to $\sim 1$ dex below, or perhaps rather to the right of, non-barred galaxies in the $M_{\mathrm{bh}}-\sigma$ diagram, with the mean vertical offset 0.3 to 0.4 dex (e.g. Graham 2008a; Hu 2008). Galaxies with AGN have also been shown to display this same behavior in the $M_{\mathrm{bh}}-\sigma$ diagram (e.g. Graham \& Li 2009, see also Wandel 2002; Mathur \& Grupe 2005; $\mathrm{Wu}$ 2009; Mathur et al. 2012, while alternate views can be found in Botte et al. 2005; Komossa \& Xu 2007; Decarli et al. 2008a and Marconi et al. 2008). This is not to say that all barred galaxies are offset from the barless $M_{\mathrm{bh}}-\sigma$ relation, only that a significant fraction are. In the analysis by $\mathrm{Hu}$ (2008), he considered the offset galaxies to be 'pseudobulges', although all of his offset galaxies were also barred galaxies. Greene, Ho, \& Barth (2008, see their figure $7 \mathrm{~b}$ ) subsequently showed that their sample of 11 'pseudobulges' was not offset from the $M_{\mathrm{bh}}$ $M_{\text {spheroid }}$ relation defined by massive classical systems ${ }^{15}$. (Oddly, their work is often mis-quoted as evidence that pseudobulges in disk galaxies are an offset population.) With new and updated data, we have observed that the

\footnotetext{
14 For clarity we note that Guo et al. (2011) excluded the square on the normalized velocity term in their eq. 36 .

15 We speculate that this lack of an offset may have been due to an underestimation of dust obscuration from the disks surrounding their bulges.
}

mean offset of (in this case 21) barred galaxies in the $M_{\mathrm{bh}}-\sigma$ diagram remains. These galaxies also appear to follow a relation which is parallel with that defined by luminous core-Sérsic galaxies. The rather narrow baseline, of effectively only $\sim 2 \mathrm{mag}$, for the 11 'pseudobulges' in Kormendy et al.'s (2011) $M_{\mathrm{bh}}-L$ diagram may partly (see also Graham 2011b) explain why they did not observe a correlation between black hole mass and bulge luminosity. It is not yet clear if barred galaxies are offset from non-barred (Sérsic) galaxies in the $M_{\mathrm{bh}}-M_{\text {spheroid }}$ diagram.

Graham (2008a) had previously noted the connection between bar instabilities and pseudobulges ${ }^{16}$, and wrote that if the offset barred galaxies have discrepantly low black hole masses rather than elevated $\sigma$-values, then they should also appear as systematic outliers in the $M_{\mathrm{bh}}-L$ diagram. Now although Graham (2007a) ${ }^{17}$, Graham \& Driver (2007a, their section 3.2) and Graham (2008b, his section 2.2.2) warned that the $M_{\mathrm{bh}}-L$ relation must be bent ${ }^{18}$, nobody had yet constructed this bent relation and it was common practice to assume that the original relation, dominated by luminous spheroids, could be extrapolated to low luminosities. However in so doing, even the classical, low-luminosity spheroids would end up incorrectly labeled because they are offset from the extrapolated relation fit to the distribution of luminous spheroids in the $M_{\mathrm{bh}}-L$ diagram (Greene, Ho, \& Barth 2008; Jiang, Greene, \& Ho 2011; Kormendy, Bender, \& Cornell 2011).

In a similar vein, and while on the discussion of pseudobulge identification, a number of recent papers on the topic of supermassive black holes have claimed the detection of pseudobulges because (due to their low Sérsic index $<2$ ) they do not follow the extrapolations of other linear approximations to the bright arms of what we now know are continuous but curved underlying distributions. This important subject of non-(log-linear) scaling relations is extensively reviewed in Graham (2012c, and references therein) and is vital if we are to properly understand the formation of spheroids. For example, semianalytical and theoretical models which still assume a log-linear size-luminosity relation (e.g. Guo et al. 2010; Suh 2012) are handicapping themselves as they are not matching galaxies in the real Universe. Galaxies which do not follow the bright arm of the curved (effective surface brightness)-(effective radius) diagram should also not be labeled as pseudobulges for use in the various black hole diagrams (e.g. Gadotti \& Kauffmann 2009; Greene et al. 2008).

Finally, we offer the following thoughts on whether or not barred galaxies might be offset in the $M_{\mathrm{bh}}-L$ diagram. Among lenticular disk galaxies, the average luminosity of the barred S0s is apparently $\sim 0.4$ mag fainter than that of the non-barred S0s, largely because there are almost no barred S0 galaxies brighter than $M_{B}=-21$ mag (van den Bergh 2012). It has additionally been re-

\footnotetext{
16 Whether or not the bulges in these galaxies formed solely from a redistribution of disk stars, to produce 'pseudobulges', is unknown due to the many difficulties in identifying pseudobulges (Graham 2011a, 2012c).

17 See the end of his Appendix A, noting that the term $L^{0.5}$ should have read $L^{2}$.

18 At this time Bernardi et al. (2007) had speculated that there may instead be curvature in the $M_{\mathrm{bh}}-\sigma$ relation
} 
ported that the average photometric bulge mass of barred galaxies is less than that of non-barred disc galaxies of similar total galaxy stellar mass (Aguerri et al. 2005; Laurikainen et al. 2007; Coelho \& Gadotti 2011, but see Laurikainen et al. 2010), and that the bulge-to-total flux ratio is smaller in barred galaxies than in non-barred galaxies of the corresponding Hubble type (Weinzirl et al. $2009)$. If the effective sizes $\left(R_{\mathrm{e}}\right)$ of bulges in barred galaxies are also smaller than those in non-barred disk galaxies, then one may find that the offset of barred galaxies toward higher velocity dispersions in the $M_{\mathrm{bh}}-\sigma$ diagram is counter-balanced when using $\sigma^{2} R_{\mathrm{e}}$ for bulge mass in the $M_{\mathrm{bh}}-M_{\text {spheroid }}$ diagram. Thus, rather than finding barred galaxies tending to reside, on average, 'rightward' of the $M_{\mathrm{bh}}-L$ relation defined by the non-barred Sérsic galaxies, the bulges of barred galaxies may yet be found to reside 'leftward' of it, i.e. 'above' it. Trying to discern if spheroids, and their black holes, in barred galaxies are offset from those of non-barred galaxies in the $M_{\mathrm{bh}}{ }^{-}$ $L$ diagram, which was the question posed by Graham (2008a), will require either a larger data sample than we currently have or reliable individual bulge/disc/bar decompositions.

\section{CONCLUSIONS}

Using a refined sample of 72 galaxies, comprised of 24 core-Sérsic galaxies plus 48 Sérsic galaxies, we have revised the $M_{\mathrm{bh}}-L_{\text {spheroid }}$ relation. We have replaced what was a single power-law relation for all spheroids with distinct relations for the core-Sérsic and the Sérsic population.

Using this exact same galaxy sample we have also updated the $M_{\mathrm{bh}}-\sigma$ relations. From 21 barred and 51 nonbarred galaxies, we confirm a mean factor of two offset between their $M_{\mathrm{bh}}-\sigma$ relations, and a steep slope of around 5 to 5.5 for the elliptical and un-barred galaxies (see Figure $2 \mathrm{a}$ and Table 3).

It remains unknown if barred galaxies are offset, to lower black hole masses or equivalently brighter luminosities, relative to the non-barred Sérsic galaxies, in the
$M_{\mathrm{bh}}-L_{\text {spheroid }}$ diagram.

The lower mass Sérsic galaxies are observed to be offset from $M_{\mathrm{bh}}-L_{\text {spheroid }}$ relation defined by the luminous galaxies. However rather than being an offset population of alleged pseudobulges, we have explained why it is instead the luminous core-Sérsic galaxies that deviate from the $M_{\mathrm{bh}}-L_{\text {spheroid }}$ relation defined by the fainter Sérsic galaxies.

Luminous core-Sérsic galaxies roughly follow the relation $M_{\mathrm{bh}} \propto L^{1}$, consistent with the concept of their formation through dry galaxy merging. Built by such simple additive merger events, rather than what were once gaseous mechanisms, a roughly constant (black hole)to-(host spheroid stellar) mass ratio of $0.35-0.55 \%$ is observed. The fainter (barred and non-barred) Sérsic galaxies follow an $M_{\mathrm{bh}}-L_{\text {spheroid }}$ relation which is some 2.5 times steeper. Thus, while the black hole mass to spheroid stellar mass ratio can be $0.5 \%$ in massive elliptical galaxies, it drops to values of $0.05 \%$ in spheroids that are not much fainter than $M_{B} \approx-19$ mag.

We have discussed the implications of this steep Sérsic $M_{\mathrm{bh}}-L_{\text {spheroid }}$ relation for the Sérsic $M_{\mathrm{bh}}-M_{\mathrm{Sph} \text {,dyn }}$ relation (Graham 2012a), which suggest that black holes grew quadratically relative to their host spheroids. We have therefore advocated a new quadratic cold-gas 'quasar' mode feeding equation (Eq. 9) for use in semianalytical models.

Finally, using our new $M_{\mathrm{bh}}-L_{\text {spheroid }}$ relation for Sérsic galaxies, we have predicted a number of intermediate mass black holes, potentially filling a substantial portion of the current observational gap between stellar mass black holes and one million solar mass black holes.

\section{ACKNOWLEDGMENT}

This research was supported under the Australian Research Councils funding scheme (DP110103509 and FT110100263).

We acknowledge use of the HyperLeda database (http://leda.univ-lyon1.fr). This research has made use of the NASA/IPAC Extragalactic Database (NED).

\section{REFERENCES}

Aguerri J.A.L., Iglesias-Páramo J., Vílchez J.M., Munoz-Tunón C., \& Sánchez-Janssen R. 2005b, AJ, 130, 475

Akritas, M.G., Bershady, M.A. 1996, ApJ, 470, 706

Atkinson, J.W., Collett, J. L., Marconi, A., et al. 2005, MNRAS, 359,504

Bacon, R., Emsellem, E., Combes, F., Copin, Y., Monnet, G., \& Martin, P. 2001, A\&A, 371, 409

Balcells, M., Graham, A., \& Peletier, R.F. 2007, ApJ, 665, 1084

Barth, A.J., Greene, J.E., \& Ho, L.C. 2005, ApJ, 619, L151

Barth, A.J., Sarzi, M., Rix, H.-W., Ho, L.C., Filippenko, A.V., \& Sargent, W.L.W. 2001, ApJ, 555, 685

Barth, A.J., Ho, L.C., Rutledge, R.E., Sargent, W.L.W. 2004, ApJ, 607,90

Batcheldor D., 2010, ApJ, 711, L108

Beifiori, A., Courteau, S., Corsini, E.M., \& Zhu, Y. 2012, MNRAS, 419, 2497

Begelman, M.C., Blandford, R.D., \& Rees, M.J. 1980, Nature, 287, 307

Bekki, K., Couch, W. J., Drinkwater, M. J., \& Gregg, M. D. 2001, ApJ, 557, L39

Bell, E. F., \& de Jong, R. S. 2001, ApJ, 550, 212

Bender R., Doebereiner S., Moellenhoff C. 1988, A\&AS, 74, 385

Bender, R., Kormendy, J., Bower, G., et al. 2005, ApJ, 631, 280

Bernardi, M., Hyde, J.B., Sheth, R.K., Miller, C.J., \& Nichol, R.C. 2007, AJ, 133, 1741

Binggeli, B., Sandage, A., \& Tarenghi, M. 1984, AJ, 89, 64
Bogdán, Á., Forman, W.R., Zhuravleva, I., et al. 2012a, ApJ, 753, 140

Bogdán, Á., Forman, W.R., Kraft, R.P., et al. 2012b, ApJ, 755, 25

Bonoli, S., Mayer, L., Callegari, S. 2012, MNRAS, submitted (arXiv:1211.3752)

Botte, V., Ciroi, S., di Mille, F., Rafanelli, P., Romano, A. 2005, MNRAS, 356, 789

Bower, G.A., Green, R. F., Bender, R., et al. 2001, ApJ, 550, 75

Blakeslee, J.P., Jordán, A., Mei, S., et al. 2009, ApJ, 694, 556

Blakeslee, J.P., Lucey, J.R., Tonry, J.L., Hudson, M.J., Narayanan, V.K., \& Barris, B.J., 2002, MNRAS, 330, 443

Blom, C., Forbes, D.A., Brodie, J.P., et al. 2012, MNRAS, 426, 1959

Bloom, J.S., Giannios, D., Metzger, B.D., et al. 2011, Science, 333, 203

Capetti, A., Marconi, A., Macchetto, D., \& Axon, D. 2005, A\&A, 431,465

Cappellari, M., Verolme, E.K., van der Marel, R.P., Kleijn, G.A.V., Illingworth, G.D., Franx, M., Carollo, C.M., \& de Zeeuw, P.T. 2002, ApJ, 578, 787

Cappellari, M., Bacon, R., Bureau, M., et al. 2006, MNRAS, 366, 1126

Cappellari, M., Bacon, R., Davies, R. L., et al. 2008, in the proceedings of IAU Symposium 245, "Formation and Evolution of Galaxy Bulges", eds. M.Bureau, E.Athanassoula, and B.Barbuy, p.215 
Cappellari, M., Emsellem, E., Krajnović, D., et al. 2011, MNRAS, 413,813

Carter, D. 1978, MNRAS, 182, 797

Carter, D. 1987, ApJ, 312, 514

Cen, R. 2012, ApJ, 755, 28

Cecil, G., Bland-Hawthorn, J., Veilleux, S., Filippenko, A.V. 2001, ApJ, 555, 338

Chilingarian, I.V., Melchior, A.-L., \& Zolotukhin, I.Y. 2010, MNRAS, 405, 1409

Cid Fernandes, R., Gu, Q., Melnick, J., et al. 2004, MNRAS, 355, 273

Cirasuolo, M., Shankar, F., Granato, G.L., De Zotti, G., Danese, L. 2005, ApJ, 629, 816

Cisternas, M., Jahnke, K., Bongiorno, A., et al. 2011, ApJ, 741, L11

Coelho, P., \& Gadotti, D.A. 2011, ApJ, 743, L13

Copin, Y., Cretton, N., \& Emsellem, E. 2004, A\&A, 415, 889

Corbin, M.R., O'Neil, E., \& Rieke, M.J. 2001, AJ, 121, 2549

Cox, D.P. 2000, Allen's Astrophysical quantities, New York: AIP Press; Springer

Cretton, N., \& van den Bosch, F. 1999, ApJ, 514, 704

Croton, D. J., Springel, V., White, S. D. M., et al. 2006, MNRAS, 365,11

Dalla Bontà E., Ferrarese L., Corsini E.M., Miralda-Escudé J., Coccato L., Sarzi M., Pizzella A., \& Beifiori A. 2009, ApJ, 690, 537

Davies, R.L., Efstathiou, G., Fall, S.M., et al. 1983, ApJ, 266, 41

Davies, R.I., Thomas, J., Genzel, R., et al. 2006, ApJ, 646, 754

DeBuhr, J., Ma, C.-P., \& White, S.D.M. 2012, MNRAS, 426, 983

Decarli, R., Dotti, M., Fontana, M., Haardt, F., 2008, MNRAS, 386, L15

de Francesco, G., Capetti, A., \& Marconi, A. 2006, A\&A, 460, 439

de Francesco, G., Capetti, A., Marconi, A. 2008, A\&A, 479, 355

DeGraf, C., Di Matteo, T., Khandai, N., \& Croft, R. 2012, ApJ, $755, \mathrm{~L} 8$

de Rijcke, S., Michielsen, D., Dejonghe, H., Zeilinger, W.W., \& Hau, G.K.T. 2005, A\&A, 438, 491

Deshmukh, S.P., Tate, B.T., Vagshette, N.D., Pandey, S.K., \& Patil, M.K. 2012, submitted to RAA (arXiv:1207.4324)

Desroches, L.-B., Quataert, E., Ma, C.-P., \& West, A. A. 2007, MNRAS, 377, 402

De Vaucouleurs, G., De Vaucouleurs, A., Corwin, H. G. Jr, Buta R. J., Paturel G., \& Fouque P. 1991, Third Reference Catalogue of Bright Galaxies. Springer-Verlag, Berlin (RC3)

de Vaucouleurs, G. \& Olson, D. W. 1982, ApJ, 256, 346

Devereux, N.A., Ford, H.C., Tsvetanov, Z., \& Jacoby, G. 2003, AJ, 125,1226

Djorgovski, S., \& Davis, M. 1987, ApJ, 313, 59

Dong, X.-B., Ho, L.C., Yuan, W., et al. 2012, ApJ, 755, 167

Dong, X.Y., \& De Robertis, M.M. 2006, AJ, 131, 1236

Driver, S. P., Popescu, C. C., Tuffs, R. J., Graham, A.W., Liske, J., \& Baldry, I. 2008, ApJ, 678, L101

Dubois, Y., Devriendt, J., Slyz, A., \& Teyssier, R. 2012, MNRAS, 420, 2662

Dullo, B., \& Graham, A.W. 2012, ApJ, submitted

Ebisuzaki, T., Makino, J., \& Okumura, S.K. 1991, Nature, 354, 212

Emsellem, E., Cappellari, M., Krajnović, D., et al. 2011, MNRAS, 414,888

Emsellem, E., Dejonghe, H., \& Bacon, R. 1999, MNRAS, 303, 495

Faber, S.M., Dressler, A., Davies, R.L., Burstein, D., LyndenBell, D., Terlevich, R., \& Wegner, G. 1987, in Nearly Normal Galaxies: From the Planck Time to the Present, edited by S.M.Faber (Springer, New York), p.175

Erwin, P., Beltrán, J.C.V., Graham, A.W., \& Beckman, J.E. 2003, ApJ, 597, 929

Faber, S.M. \& Jackson, R.E. 1976, ApJ, 204, 668

Faber, S.M., Tremaine, S., Ajhar, E.A., et al. 1997, AJ, 114, 1771

Falcke, H., Körding, E., Markoff, S. 2004. A\&A, 414, 895

Falcón-Barroso, J., van de Ven, G., Peletier, R.F., et al. 2011, MNRAS, 417, 1787

Farrell, S.A., Webb, N.A., Barret, D., Godet, O., \& Rodrigues, J.M. 2009, Nature, 460, 73

Ferrarese, L., Freedman, W. L., Hill, R. J., et al. 1996, ApJ, 464, 568

Ferrarese, L. 2002, ApJ, 578, 90

Ferrarese, L., Côté, P., Jordán, A., et al. 2006, ApJS, 164, 334

Ferrarese, L., \& Ford, H.C. 1999, ApJ, 515, 583

Ferrarese, L., \& Ford, H.C. 2005, Space Science Reviews, 116, 523

Ferrarese, L.A., \& Merritt, D. 2000, ApJ, 539, L9
Ferrarese, L., van den Bosch, F.C., Ford, H.C., Jaffe, W., \& O'Connell, R.W. 1994, AJ, 108, 1598

Filippenko, A.V., \& Ho, L.C. 2003, ApJ, 588, L13

Fisher, D.B., Drory, N. 2008, AJ, 136, 773

For, B.-Q., Koribalski, B., Jarrett, T. 2012, MNRAS, 425, 1934

Freedman, W. L., Madore, B. F., Gibson, B. K., et al. 2001, ApJ, 553,47

Gadotti D.A., \& Kauffmann G. 2009, MNRAS, 399, 621

Gavazzi, G., Donati, A., Cucciato, O., Sabatini, S., Boselli, A., Davies, J., \& Zibetti, S. 2005, A\&A, 430, 411

Gebhardt, K., Adams, J., Richstone, D., et al. 2011, ApJ, 729, 119

Gebhardt, K., Richstone, D., Tremaine, S., et al. 2003, ApJ, 583, 92

Gebhardt, K., Bender, R., Bower, G., et al. 2000, ApJ, 539, L13

Gebhardt, K., Lauer, T. R., Pinkney, J., et al. 2007, ApJ, 671, 1321

Gerssen, J., Kuijken, K., \& Merrifield, M.R. 1999, MNRAS, 306, 926

Gezari, S., Chornock, R., Rest, A., et al. 2012, Nature, 485, 217

Gillessen, S., Eisenhauer, F., Fritz, T.K., Bartko, H., Dodds-Eden, K., Pfuhl, O., Ott, T., Genzel, R. 2009, ApJ, 707, L114

González Delgado, R.M., Pérez, E., Cid Fernandes, R., \& Schmitt, H. 2008, AJ, 135, 747

Graham, A.W. 2002, ApJ, 568, L13

Graham, A.W. 2004, ApJ, 613, L33

Graham, A.W. 2007b, BAAS, 39759 \#13.27

Graham, A.W. 2007a, MNRAS, 379, 711

Graham, A.W. 2008a, ApJ, 680, 143

Graham, A.W. PASA, 2008b, 25, 167

Graham, A.W. 2011a, in "A Universe of dwarf galaxies", EAS Pub. Ser., 48, 231 (arXiv:1009.5002)

Graham, A.W. 2011b, (arXiv:1103.0525)

Graham, A.W. 2012a, ApJ, 746, 113

Graham, A.W. 2012b, MNRAS, 422, 1586

Graham, A.W. 2012c, in "Planets, Stars and Stellar Systems", Springer Publishing (arXiv:1108.0997)

Graham, A.W., \& Driver, S.P. 2005, PASA, 22(2), 118

Graham, A.W., \& Driver, S.P. 2007a, ApJ, 655, 77

Graham, A.W., \& Driver, S.P. 2007b, MNRAS, 380, L15

Graham, A.W., Erwin, P., Trujillo, I., \& Asensio Ramos, A. 2003, AJ, 125, 2951

Graham, A.W. \& Guzmán, R. 2003, AJ, 125, 2936

Graham, A.W., \& Li, I-H. 2009, ApJ, 698, 812

Graham, A.W., Onken, C., Athanassoula, L., \& Combes, F. 2011, MNRAS. 412, 2211

Graham, A. W., \& Spitler, L. R. 2009, MNRAS, 397, 2148

Graham, A. W., \& Worley, C. C. 2008, MNRAS, 388, 1708

Greene, J.E., Ho, L.C., \& Barth, A.J. 2008, ApJ, 688, 159

Greene, J.E., Zakamska, N.L., Liu, X., Barth, A.J., \& Ho, L.C. 2009, ApJ, 702, 441

Greene, J.E., Peng, C.Y., Kim, M., et al. 2010, ApJ, 721, 26

Greenhill, L.J., Moran, J.M., \& Herrnstein, J.R. 1997, ApJ, 481, L23

Greenhill, L.J., Booth, R. S., Ellingsen, S. P., et al. 2003, ApJ, 590, 162

Grillmair, C.J., Faber, S.M., Lauer, T.R., et al. 1994, AJ, 108, 102

Grootes, M.W., Tuffs, R.J., Popescu, C.C., et al. MNRAS, in press

Gültekin K., Richstone, D. O., Gebhardt, K., et al. 2009a, ApJ, 695,1577

Gültekin K., Richstone, D. O., Gebhardt, K., et al. 2009b, ApJ, 698, 198

Gültekin, K., Richstone, D.O., Gebhardt, K., et al. 2011, ApJ, 741, 38

Guo, Q., White, S., Boylan-Kolchin, M., et al. 2011, MNRAS, 413, 101

Häring, N. \& Rix, H.-W. 2004, ApJ, 604, L89

Held, E. V., de Zeeuw, T., Mould, J., \& Picard, A. 1992, AJ, 103, 851

Herrnstein, J.R., Moran, J. M., Greenhill, L. J., et al. 1999, Nature, 400,539

Hernández-Toledo, H. M., Cano-Díaz, M., Valenzuela, O., et al. 2011, AJ, 142, 182

Herrmann, K. A., Ciardullo, R., Feldmeier, J. J., \& Vinciguerra, M. 2008, ApJ, 638, 630

Hicks, E.K.S., \& Malkan, M.A. 2008, ApJS, 174, 31

Hiner, K.D., Canalizo, G., Wold, M., Brotherton, M.S., \& Cales, S.L. 2012, ApJ, 756, 162

Hirschmann, M., Somerville, R.S., Naab, T., Burkert, A. 2012, MNRAS, 426, 237

Hlavacek-Larrondo, J., Fabian, A.C., Edge, A.C., Hogan, M.T. 2012, MNRAS, 424, 224 
Ho, L.C. 1999, in Observational Evidence for Black Holes in the Universe, ed. S.K. Chakrabarti (Dordrecht: Kluwer), 157

Ho, L.C. 2007, ApJ, 668, 94

Ho, L.C., Greene, J.E., Filippenko, A.V., \& Sargent, W.L.W. 2009, ApJS, 183, 1

Hopkins, P.F., Hernquist, L., Cox, T.J., et al. 2006, ApJS, 163, 1

Houghton, R.C.W., Magorrian, J., Sarzi, M., Thatte, N., Davies, R.L., \& Krajnović, D. 2006, MNRAS, 367, 2

Hu, J. 2008, MNRAS, 386, 2242

Ishihara, Y., Nakai, N., Iyomoto, N., Makishima, K., Diamond, P., \& Hall, P. 2001, PASJ, 53, 215

Jaffe, W., Ford, H.C., O'Connell, R.W., van den Bosch, F.C., Ferrarese, L. 1994, AJ, 108, 1567

Jahnke, K., \& Macciò, A. V. 2011, ApJ, 734, 92

Jardel, J.R., Gebhardt, K., Shen, J., et al. 2011, ApJ, 739, 21

Jarrett, T.H., Chester, T., Cutri, R., et al. 2000, AJ, 119, 2498 (2MASS)

Jerjen, H., Binggeli, B., \& Barazza, F.D. 2004, AJ, 127, 771

Jiang, Y.-F., Greene, J.E., \& Ho, L.C. 2011, ApJ, 737, L45

Jiménez, N., Cora, S.A., Bassino L.P., Tecce T.E., \& Smith Castelli A.V. 2011, MNRAS, 417, 785

Johansson, P.H., Naab, T., Burkert, A. 2009, ApJ, 690, 802

Karachentsev, I.D., Tully, R. B., Dolphin, A., et al. 2007, AJ, 133, 504

Kauffmann, G., \& Haehnelt, M. 2000, MNRAS, 311, 576

Khandai, N., Feng, Y., DeGraf, C., Di Matteo, T.,Croft, R.A.C. 2012, MNRAS, 423, 2397

Khorunzhev, G.A., Sazonov, S.Y., Burenin, R.A., Tkachenko, A.Y. 2012, Astronomy Letters, 38(8), 475

King I.R. 1978, ApJ, 222, 1

Kisaka, S., \& Kojima, Y. 2010, MNRAS, 405, 1285

Knapen, J.H., de Jong, R.S., Stedman, S., \& Bramich, D.M. 2003, MNRAS, 344, 527

Komossa S., Xu D., 2007, ApJ 667, L33

Kondratko, P.T., Greenhill, L.J., \& Moran, J.M. 2005, ApJ, 618, 618

Kondratko, P.T., Greenhill, L.J., \& Moran, J.M. 2008, ApJ, 678, 87

Kormendy, J. 1985, ApJ, 295, 73

Kormendy, J., Bender, R., \& Cornell, M.E. 2011, Nature, 469, 374

Kormendy, J., Fisher, D.B., Cornell, M.E., \& Bender, R. 2009, ApJS, 182, 216

Kormendy, J., \& Gebhardt, K., in H.Martel, J.C.Wheeler, eds, 2001 20th Texas Symposium on relativistic astrophysics, Am. Inst. Phys., New York, Conf. Proc., 586, 363

Kormendy, J., \& Richstone, D. 1995, ARA\&A, 33, 581

Kornei, K.A., \& McCrady, N. 2009, ApJ, 697, 1180

Kourkchi, E., Khosroshahi, H. G., Carter, D., et al., 2012, MNRAS, 420, 2819

Krajnović D., McDermid R.M., Cappellari M., \& Davies R.L. 2009, MNRAS, 399, 1839

Kunth, D., Sargent, W.L.W., Bothun, G.D. 1987, AJ, 93, 29

Kuo, C.Y., Braatz, J.A., Condon, J.J., et al. 2011, ApJ, 727, 20

La Barbera, F., de Carvalho, R.R., de La Rosa, I.G., \& Lopes, P.A.A. 2010, MNRAS, 408, 1335

Lahav, C.G., Meiron, Y., Soker, N. 2011 (arXiv:1112.0782)

Laine, S., van der Marel, R.P., Lauer, T.R., et al. 2003, AJ, 125, 478

Laor, A. 2001, ApJ, 553, 677

Lauer, T.R., Faber, S. M., Gebhardt, K., et al. 2005, AJ, 129, 2138

Lauer, T.R., Faber, S. M., Richstone, D., et al. 2007, ApJ, 662, 808

Laurikainen, E., Salo, H., \& Buta, R. 2005, MNRAS, 362, 1319

Laurikainen, E., Salo, H., Buta, R., \& Knapen, J. H. 2007, MNRAS, 381,401

Laurikainen, E., Salo, H., Buta, R., Knapen, J. H., \& Comerón, S. 2010, MNRAS, 405, 1089

Laurikainen, E., Salo, H., Buta, R., \& Knapen, J. H. 2011, MNRAS, 418,1452

Leigh, N., Böker, T., \& Knigge, C. 2012, MNRAS, 424, 2130

Levan, A.J., Tanvir, N.R., Cenko, S.B., et al. 2011, Science, v.333, no.6039, p.199

Li, Y.-R., Ho, L.C., \& Wang, J.-M. 2011, ApJ, 742, 33

Li, Y.-R., Wang, J.-M., \& Ho, L.C. 2012, IAU Symp. 290, C.M. Zhang, T. Belloni, M. Mendez \& S.N. Zhang (eds.)

Liu, F.S., Xia, X.Y., Mao, S., Wu, H., \& Deng, Z.G. 2008, MNRAS, 385,23

Lodato, G., \& Bertin, G. 2003, A\&A, 398, 517

Lynden-Bell D., Faber S.M., Burstein D., Davies R.L., Dressler A., Terlevich R.J., \& Wegner G. 1988, ApJ, 326, 19
Macchetto, F., Capetti, A., Sparks, W.B., Axon, D.J., \& Boksenberg, A. 1994, ApJ, 435, L15

Madore B.F., Freedman, W. L., Silbermann, N., et al., 1999, ApJ, 515,29

Magorrian, J., Tremaine, S., Richstone, D., et al., 1998, AJ, 115, 2285

Magoulas, C., Springob, C.M., Colless, M.M., et al. 2012, MNRAS, in press (arXiv:1206.0385)

Malkan, M.A., Gorjian, V., \& Tam, R. 1998, ApJS, 117, 25

Malumuth, E.M., \& Kirshner. R.P. 1981, ApJ, 251, 508

Mapelli M., Ripamonti E., Vecchio A., Graham A.W., \& Gualandris A. 2012, A\&A, 542, 102

Marconi, A., Axon, D.J., Maiolino, R., Nagao, T., Pastorini, G., Pietrini, P., Robinson, A., Torricelli, G., 2008, ApJ, 678, 693

Marconi, A., \& Hunt, L.K. 2003, ApJ, 589, L21

Marconi, A., Oliva, E., van der Werf, P.P., et al. 2000, A\&A, 357, 24

Mathur, S., Fields, D., Peterson, B.M., \& Grupe, D. 2012, ApJ, 754,146

Mathur S., Grupe D., 2005, ApJ, 633, 688

Matković, A., \& Guzmán, R. 2005, MNRAS, 362, 289

McAlpine, W., Satyapal, S., Gliozzi, M., et al. 2011, ApJ, 728, 25

McConnell, N.J., Ma, C.-P., Gebhardt, K., et al. 2011a, Nature, 480, 215

McConnell, N.J., Ma, C.-P., Graham, J.R., et al. 2011b, ApJ, 728, 100

McConnell, N.J., Ma, C.-P., Murphy J.D., et al. 2012, ApJ, 756 179

McLure R. J., \& Dunlop J. S. 2002, MNRAS, 331, 795

McLure R. J., \& Dunlop J. S. 2004, MNRAS, 352, 1390

Mei, S., Blakeslee, J.P., \& Côté, P., et al. 2007, ApJ, 655, 144

Merloni, A., Heinz, S., \& di Matteo, T. 2003, MNRAS, 345, 1057

Merrifield, M.R., \& Kuijken, K. 1999, A\&A, 345, L47

Merritt D., Ferrarese L., 2001a, ApJ, 547, 140

Merritt D., \& Ferrarese L. 2001b, in The Central Kiloparsec of Starbursts and AGN: The La Palma Connection, ASP Conference Proceedings Vol. 249, J.H. Knapen, J.E. Beckman, I. Shlosman, and T.J. Mahoney eds., San Francisco: Astronomical Society of the Pacific, p.335.

Merritt, D., Mikkola, S., \& Szell, A. 2007, ApJ, 671, 53

Muñoz Marín, V.M., González Delgado, R.M., Schmitt, H.R., et al. 2007, AJ, 134, 648

Nakai, N., Inoue, M., Hagiwara, Y., Miyoshi, M., \& Diamond, P.J. 1998, IAU Colloq. 164: Radio Emission from Galactic and Extragalactic Compact Sources, 144, 237

Nelson, C.H., \& Whittle, M. 1995, ApJS, 99, 67

Neumayer, N., 2010, Pub. Astron. Soc. Aust., 27, 449

Neumayer, N., \& Walcher, C.J. 2012, Advances in Astronomy, 2012 , id. 709038

Nieto, J.-L., Bender, R., \& Surma, P. 1991, A\&A, 244, L37

Nowak, N., Saglia, R.P., Thomas, J., Bender, R., Davies R.I., \& Gebhardt K. 2008, MNRAS, 391, 1629

Nowak, N., Saglia, R.P., Thomas, J., Bender, R., Pannella, M., Gebhardt, K., \& Davies, R.I. 2007, MNRAS, 379, 909

Nowak N., Thomas J., Erwin P., Saglia R.P., Bender R., \& Davies R.I. 2010, MNRAS, 403, 646

Nyland, K., Marvil, J., Wrobel, J.M., Young, L.M., \& Zauderer, B.A. 2012, ApJ, 753, 103

Oliva E., Origlia L., Kotilainen J.K., \& Moorwood A.F.M. 1995, A\&A 301, 55

Onken, C.A., Ferrarese, L., Merritt, D., et al. 2004, ApJ, 615, 645

Onken, C.A., Valluri, M., Peterson, B. M., et al. 2007, ApJ, 670, 105

Park, D., Kelly, B.C., Woo, J.-H., Treu, T. 2012, ApJS, 203, 6

Pastorini, G., Marconi, A., Capetti, A., et al. 2007, A\&A, 469, 405

Paturel G., Petit C., Prugniel P., Theureau G., Rousseau J., Brouty M., Dubois P., \& Cambrésy L. 2003, A\&A, 412, 45

Peletier, R.F., Davies, R.L., Illingworth, G.D., Davis, L.E., Cawson, M. 1990, AJ, 100, 1091

Peng, C.-Y. 2007, ApJ, 671, 1098

Peterson, B.M., Bentz, M.C., Desroches, L.-B., et al. 2005, ApJ, 632, 799

Pogge, R.W., \& Martini, P. 2002, ApJ, 569, 624

Popescu, C.C., Misiriotis, A., Kylafis, N.D., Tuffs, R.J., \& Fischera, J. 2000, A\&A, 362, 138

Portinari, L., Kotilainen, J., Falomo, R., \& Decarli, R. 2012, MNRAS, 420, 732

Quillen, A. C., Bower, G. A., \& Stritzinger, M. 2000, ApJS, 128, 85 
Radomski, J.T., Packham, C., Levenson, N.A., et al. 2008, ApJ, 681, 141

Ravindranath, S., Ho, L., Peng, C., et al. 2001, AJ, 122, 653

Rekola R., Richer M.G., McCall M.L., Valtonen M.J., Kotilainen J.K., \& Flynn C. 2005, MNRAS, 361, 330

Rest, A., van den Bosch, F.C., Jaffe, W., et al. 2001, 121, 2431

Richings, A.J., Uttley, P. and Kórding, E. 2011, MNRAS, 415, 2158

Richstone, D., Ajhar, E.A., Bender, R., et al. 1998, Nature, 395, A14

Rix, H.-W., Carollo, C.M., \& Freeman, K. 1999, ApJ, 513, L25

Rodríguez-Rico, C.A., Goss, W.M., Zhao, J.-H., Gómez, Y., \& Anantharamaiah, K.R. 2006, ApJ, 644, 914

Rusli, S.P., Thomas, J., Erwin, P., et al. 2011, MNRAS, 410, 1223

Sanders, D.B., \& Mirabel, I.F. 1996, ARA\&A, 34, 749

Sani, E., Marconi, A., Hunt, L.K., \& Risaliti, G. 2011, MNRAS, 413, 1479

Sarzi, M., Rix, H.-W., Shields, J.C., Rudnick, G., Ho, L.C., McIntosh, D.H., Filippenko, A.V., \& Sargent, W.L.W. 2001 ApJ, 550, 65

Schechter, P.L. 1980, AJ, 85, 801

Schlafly, E.F., \& Finkbeiner, D.P. 2011, ApJ, 737, 103

Schlegel, D.J., Finkbeiner, D.P., \& Davis, M. 1998, ApJ, 500, 525

Schombert, J., 2011, arXiv:1107.1728

Schombert, J., \& Smith, A.K. 2012, PASA, PASA, 29, 174

Schulze, A., \& Wisotzki, L. 2011, A\&A, 535, A87

Scott, N., \& Graham, A.W. 2012, ApJ, submitted (arXiv:1205.5338)

Sérsic, J.-L. 1963, Boletin de la Asociacion Argentina de Astronomia, vol.6, p.41

Sesana, A. 2012, Advances in Astronomy, vol. 2012, id.\#805402 (arXiv:1110.6445)

Seth, A., Agüeros, M., Lee, D., Basu-Zych, A. 2008, 678, 116

Seth, A.C., Cappellari, M., Neumayer, N., et al. 2010, ApJ, 714, 713

Seymour, N., Altieri, B., De Breuck, C., et al. 2012, ApJ, 755, 146

Shankar, F., Marulli, F., Mathur, S., Bernardi, M., \& Bournaud, F. 2012, A\&A, 540, A23

Shaw, M.A., Wilkinson, A., \& Carter, D. 1993, A\&A, 268, 511

Shen, J., \& Gebhardt, K. 2010, ApJ, 711, 484

Shields, J.C., Walcher, C.J., Böker, T., et al. 2008, ApJ, 682, 104

Smith, R.J., Lucey, J.R., Hudson, M.J., Schlegel, D.J., \& Davies R.L. 2000, MNRAS, 313, 469

Soria, R., Hau, G.K.T., Graham, A.W., Kong, A.K.H., Kuin, N.P.M., Li, I.-H., Liu, J.-F. \& Wu, K. 2010, MNRAS, 405, 870

Suh, P.K. 2012, International Journal of Astronomy and Astrophysics, 2, 101

Sutton, A. D., Roberts, T. P., Walton, D. J., Gladstone, J.C., Scott, A.E., 2012, MNRAS, 423, 1154

Tadhunter, C., Marconi, A., Axon, D., Wills, K., Robinson, T.G., \& Jackson, N. 2003, MNRAS, 342, 861

Tonry, J. 1981, ApJ, 251, L1
Tonry, J. L., Dressler, A., Blakeslee, J. P., et al. 2001, ApJ, 546, 681

Tremonti, C.A., Heckman, T.M., Kauffmann, G., et al. 2004, ApJ, 613,898

Trotter, A. S., Greenhill, L. J., Moran, J. M., Reid, M. J., Irwin, J. A., \& Lo, K. 1998, ApJ, 495, 740

Trujillo, I., Asensio Ramos, A., Rubiño-Martin, J.A., Graham, A.W., Aguerri, J.A.L., Cepa, J., Gutiérrez, C.M. 2002, MNRAS, 333,510

Trujillo, I., Erwin, P., Asensio Ramos, A., \& Graham, A.W. 2004, AJ, 127,1917

Tuffs, R.J., Popescu, C.C., Völk, H.J., Kylafis, N.D., \& Dopita, M.A. 2004, A\&A, 419, 821

Valluri, M., Merritt, D., \& Emsellem, E. 2004, ApJ, 602, 66

Valtonen, M.J., Ciprini, S. \& Lehto, H.J. 2012, MNRAS, 427, 77

van den Bergh, S. 2012, ApJ, 754, 68

van den Bosch R.C.E., \& de Zeeuw P.T. 2010, MNRAS, 401, 1770

van den Bosch, F.C., Jaffe, W., \& van der Marel, R.P. 1998, MNRAS, 293, 343

van der Marel, R.P., \& van den Bosch, F.C. 1998, AJ, 116, 2220

Vaughan, S., Iwasawa, K., Fabian, A.C., Hayashida, K. 2005, MNRAS, 356, 524

Veilleux, S., Bland-Hawthorn, J., \& Cecil, G. 1999, AJ, 118, 2108

Verolme, E.K., et al. 2002, MNRAS, 335, 517

Vika, M., Driver, S. P., Cameron, E., Kelvin, L., \& Robotham, A. 2011, MNRAS, 419, 2264

Vika, M., Driver, S., Graham, A., \& Liske, J. 2009, MNRAS, 400, 1451

Villar-Martín, M., Cabrera Lavers, A., Bessiere, P., Tadhunter, C., Rose, M. \& de Breuck, C. 2012, MNRAS, 423, 80

Violette Impellizzeri, C.M., Braatz, J.A., Kuo, C.-Y., Reid, M.J., Lo, K.Y., Henkel, C., \& Condon, J.J. 2012, in "Cosmic Masers- from OH to H0", Cambridge University Press, R.S. Booth, E.M.L. Humphreys \& W.H.T. Vlemmings, eds., IAU Symposium 287, 311

von der Linden, A., Best, P.N., Kauffmann, G., \& White, S.D.M. 2007, 379, 867

Walsh, J.L., Barth, A.J., \& Sarzi, M. 2010, ApJ, 721, 762

Walsh, J.L., van den Bosch, R.C.E., Barth, A.J., et al. 2012, ApJ, 753,79

Wandel A., 2002, ApJ, 565, 762

Weinzirl, T., Jogee, S., Khochfar, S., et al. 2009, ApJ, 696, 411

Wold, M., Lacy, M., Käufl, H.U., \& Siebenmorgen, R. 2006, A\&A, 460,449

Wold, M., \& Galliano, E. 2006, MNRAS, 369, L47

Wu Q., 2009, MNRAS, 398, 1905

Yamauchi, A., Nakai, N., Sato, N., \& Diamond, P. 2004, PASJ, 56, 605

Yoshizawa, M., \& Wakamatsu, K. 1975, A\&A, 44, 363

Zhang, X., Lu, Y., Yu, Q. 2012, ApJ, 761, 5 (arXiv:1210.4019) 